\title{
Fetal Transplants Alter the Development of Function after Spinal Cord Transection in Newborn Rats
}

\author{
Dorene Miya, ${ }^{1}$ Simon Giszter, ${ }^{1}$ Futoshi Mori, ${ }^{2}$ Vijayalakshmi Adipudi, ${ }^{1}$ Alan Tessler, ${ }^{3}$ and Marion Murray ${ }^{1}$ \\ ${ }^{1}$ Department of Neurobiology and Anatomy, Allegheny University of the Health Sciences, Philadelphia, Pennsylvania \\ 19129, ${ }^{2}$ Department of Neurosurgery, Akita University School of Medicine, Akita City, Akita 010, Japan, and ${ }^{3}$ The \\ Philadelphia Veteran's Administration Medical Center, Philadelphia, Pennsylvania 19104
}

Pieces of fetal spinal tissue were transplanted into the site of complete midthoracic spinal transections in neonatal rat pups (transplant rats). The development of locomotion in these animals was compared with that of unoperated control rats and rats that received spinal transections alone (spinal rats). Reflex, treadmill and overground locomotion, staircase descent, and horizontal ladder crossing for a water reward were tested in control, spinal, and transplant rats from 3 weeks to adulthood. All tests were readily performed by control animals. Most spinal rats were unable to make many linked weight-supported steps on these tasks. Transplant rats were variable in their locomotor capabilities, but a subset of rats were able to demonstrate coordinated and adaptable locomotion on these tasks. Some transplant rats performed better on more challenging tasks, suggesting that motor strategies for these tasks used different information, perhaps from descending systems. Transplanted tissue survived, and in most cases there was immunocytochemical staining of serotonergic fibers passing into and caudal to the transplant, supporting the conclusion that descending systems grew through the transplanted tissue. Integration with the host tissue was often poor, suggesting that nonspecific or trophic effects of the transplant might also contribute to the development of locomotor function. Therefore several mechanisms may contribute to the repair of injured spinal cord provided by transplants that permit the development of useful locomotion.

Keys words: spinal transection; fetal transplants; recovery of function; reflex locomotion; treadmill; overground locomotion
Embryonic spinal transplants placed into the site of a complete midthoracic spinal transection in kittens permit development of locomotion that exceeds that of littermates with transections alone (Howland et al., 1995b,c). The locomotor performance of neonatal spinal rats given transplants (Iwashita et al., 1994) also seems to exceed that in spinal rats (Stelzner et al., 1975; Weber and Stelzner, 1977). Mechanisms by which transplants mediate development of coordinated locomotion include providing an environment that supports growth of axotomized and latedeveloping axons (Bregman and Kunkel-Bagden, 1988; Howland et al., 1995c) and rescue of neurons destined to undergo retrograde cell death (Bregman and Reier, 1986; Himes et al., 1994; Mori et al., 1997). Transplants therefore may restore some elements of spinal circuitry or otherwise effect a reorganization of the host tissue (Dunnett and Bjorklund, 1994; Kargo et al., 1996) (S. Giszter and W. Kargo, unpublished observations) that is sufficient to mediate function. Because a spinal lesion sparing small amounts of tissue permits considerable function (Blight and DeCrescito, 1986), it is reasonable to expect that function can be

Received Sept. 23, 1996; revised March 21, 1997; accepted April 3, 1997.

This research was supported by National Institute of Neurological Disorders and Stroke Grant NS24707 from National Institutes of Health, The American Paralysis Association, the International Foundation for Research in Paraplegia, the Paralyzed Veterans of America, The Veteran's Administration Medical Research Service, and Allegheny Health Education and Research Foundation. Major technical contributions were made by Theresa Connors, Tim Himes, Adam Elliot, Steve Fehrer, and

Kathy Bozek. We also thank our colleagues Itzhak Fischer, Don Faber, and Kenny Simansky for their helpful discussions.

Correspondence should be addressed to Marion Murray, Department of Neurobiology and Anatomy, Allegheny University of the Health Sciences, 3200 Henry Avenue, Philadelphia, PA 19129.

Copyright (C) 1997 Society for Neuroscience $\quad 0270-6474 / 97 / 174856-17 \$ 05.00 / 0$ achieved when the spinal cord is repaired by a transplant, even without full restitution of the normal pathways.

Analysis of the motor behavior can distinguish ways in which animals with transplants differ from normal or spinal animals and clarify the mechanisms by which transplants enhance locomotor performance. The evaluation of development or recovery after spinal cord injury has usually involved either spontaneous behavior (Weber and Stelzner, 1977; Iwashita et al., 1994) or noncomplex motivated locomotor tasks (Kunkel-Bagden et al., 1993). Analysis of spontaneous behaviors such as locomotion on grids and inclined planes and in the open field can document recovery yet may provide only limited insight into the mechanisms responsible. Impaired rats may adopt different behavioral strategies that use various neural pathways; this is an especially important confounding factor when incomplete lesions are used. Complete transections provide unambiguous lesions that produce qualitative and quantifiable motor deficits. The use of a battery of conditioned motor tasks increases the uniformity of the responses and reveals both the specific details of the deficits and the maximum extent of recovery (Barbeau and Rossignol, 1987; Goldberger et al., 1990; Edgerton et al., 1992; Pratt et al., 1994; Macpherson et al., 1997).

In this study, we describe the performance of normal, spinal, and transplanted rats trained on tasks that challenged components of locomotion. We assessed the contribution of the transplant by retransecting the cord rostral to the transplant in some animals. Our results indicate that fetal transplants placed into the site of a complete transection in newborns survive and integrate at least partially with the host spinal cord. Transplants permit animals to develop overground locomotion characterized by weight support, flexible postural adjustments, and interlimb coordination 


\begin{tabular}{lllll}
\hline Table 1. Summary of experimental procedures & & \\
E14 & P0-P2 & P7 & P21 & P35, P42 \\
\hline $\begin{array}{l}\text { Fetal tissue } \\
\text { harvested } \\
\text { from donor }\end{array}$ & $\begin{array}{c}\text { Transection, } \\
\text { transplantation } \\
\text { of fetal tissue }\end{array}$ & Pretraining & $\begin{array}{c}\text { Weaning, } \\
\text { training } \\
\text { begin }\end{array}$ & $\begin{array}{c}\text { Testing } \\
\text { sessions }\end{array}$ \\
\hline
\end{tabular}

that is superior to that developed by spinal animals. Furthermore, some of these animals perform more precisely on more challenging tasks than on less challenging tasks, suggesting that they can use information from descending systems to modify their locomotion but do so only when it is necessary to achieve the goal.

\section{MATERIALS AND METHODS}

\section{Subjects}

Sprague Dawley rats were used. The experimental paradigm is outlined in Table 1. Female pups were obtained within $48 \mathrm{hr}$ of birth. Littermates were assigned to unoperated control (control, $n=6$ ), spinal transection (spinal, $n=11$ ), and transection plus transplantation (transplant, $n=11$ ) groups. Litters were culled so that only two unoperated pups remained with the mother to decrease competition among the pups and increase the likelihood of survival of the operated pups. At $7 \mathrm{~d}$ of age, pups were separated from their mother and brought to the training room for $1 \mathrm{hr}$ each day to be weighed, handled, and observed before formal training started at $21 \mathrm{~d}$ of age. This pretraining schedule seemed to increase survival and facilitate behavioral training. All operated and unoperated pups were handled and treated identically except for the surgical procedures. All procedures were performed under the guidelines of National Institutes of Health and approved by the Institutional Animal Care and Utilization Committee.

\section{Surgical procedures}

Spinal transection. Pups were anesthetized by hypothermia. Under a dissecting microscope, the spinal cord was exposed by partial laminectomy at the T8-9 level. The dura was slit, and the spinal cord was severed completely by iridectomy scissors followed by aspiration. This operation created a cavity and removed up to two segments of spinal cord. The completeness of the transection was confirmed visually and by the absence of movement of the rostral stump when the caudal spinal cord was moved gently. The gap between the spinal stumps was filled with gelfoam. The site of the transection was covered with Durafilm, and the muscle and skin were sutured in layers with 5-0 sutures. The head and flanks of operated pups were coated with peanut oil to distract the mother from the sutures. Animals were warmed and placed back with their mother and littermates when they became active.

Fetal transplantation. Tissue from embryonic day 14 fetuses was obtained from timed pregnant rats. The dams were anesthetized with an intraperitoneal injection of a cocktail of ketamine hydrochloride (95 $\mathrm{mg} / \mathrm{kg})$, xylazine $(10 \mathrm{mg} / \mathrm{kg})$, and acepromazine $(0.7 \mathrm{mg} / \mathrm{kg})$, and laparotomized. One investigator removed the spinal cords from the fetuses and dissected off the meninges. The tissue to be transplanted, which contained both cervical and thoracic regions of the cord, was cut transversely into $2-\mathrm{mm}$-thick pieces and placed in a covered Petri dish containing DMEM on ice. At the same time, a second investigator transected the spinal cord in neonatal host animals, using the method described above but omitting the insertion of gelfoam. One or two whole pieces of fetal spinal cord were inserted between the spinal stumps, and the dura was replaced over the transplantation site and covered with Durafilm. Attempts were made to maintain rostrocaudal and dorsoventral orientation of the transplanted tissue, but this could not be assured.

\section{Behavioral training}

For the first 3 weeks, pups remained with their mothers and littermates. Observations on the development of motor behavior were made in animals from three of the litters during the second and third postnatal weeks. The time required for righting responses was recorded. At 3 weeks of age, all pups were weaned and housed two per cage under a $12 \mathrm{hr}$ light/dark cycle and placed on a schedule of limited water availability. Animals were allowed access to $10 \%$ sucrose solution from the spout at the treadmill in the training room and to water in their home cages for 1
Table 2. Roster of animals

\begin{tabular}{lclll} 
Group/Test & $\begin{array}{l}\text { Quadrupedal } \\
\text { treadmill }\end{array}$ & $\begin{array}{l}\text { Wide and } \\
\text { narrow } \\
\text { runways }\end{array}$ & $\begin{array}{l}\text { Stair } \\
\text { descent }\end{array}$ & $\begin{array}{l}\text { Horizontal } \\
\text { ladder }\end{array}$ \\
\hline Control & 6 & 4 & 6 & 4 \\
Spinal & 7 & 4 & 7 & 5 \\
Transplant & 10 & 8 & 8 & 8 \\
\hline
\end{tabular}

$\mathrm{hr}$ thereafter. Animals were weighed twice daily; those gaining $<5 \mathrm{gm} /$ session were given supplemental water.

Animals used for the formal behavioral training belonged to litters that contained at least two surviving transplant animals, one spinal animal, and one unoperated control. In addition, operated animals were assessed for general health by consistent weight gain and the ability to generate righting reflexes during the preweaning period.

During the training period of 5-10 weeks, animals were trained 3-5 $\mathrm{d} /$ week to walk on a treadmill, cross wide and narrow runways, descend stairs, and cross a horizontal ladder for a water reward that was dispensed manually by the drop. A minimum of four animals per group were tested on each behavioral test (Table 2). In each test, the rats could achieve the reward by locomotion using the forelimbs only, and therefore they were not penalized for failure to use their hindlimbs. The trainers were aware of the surgical history during training of most, but not all, animals. After each training session, animals were allowed $60 \mathrm{~min}$ of free access to water. Rats were maintained on this limited water schedule for the duration of the experiment.

Locomotor performance was videotaped in two or three orthogonal (lateral, ventral, and in some cases dorsal) views. Data were collected at $30 \mathrm{~Hz}$ from a Panasonic video camera (shutter speed 1/1000 sec, $30 \mathrm{~Hz}$ frame rate at 60 fields/sec). Rats were videotaped from a distance of 12 feet to minimize perspective distortions. A Sony video camera (shutter speed $1 / 1000 \mathrm{sec}$ ) was used to film ventral views of the animals traversing the middle 14 inches of the runways. Lateral and ventral views were analyzed frame by frame using a PC-based frame grabber and software package that we developed for this study. With this system, an image is frozen when the entire body axis of the rat is on the screen. Frames in which a step was taken were frozen. The body axis and the axes of all placed feet were digitized from the base of the foot to the end of the middle phalange. The position and phase of stepcycle of all other limbs were recorded. In the rat, the commonly used subphases in the step cycle (E1, E2, E3, F) are difficult to assess reliably from videotapes; therefore, we recorded only whether the limbs were in the swing $(F)$ or stance (E) phase. Videotapes were evaluated by two observers; quantitative data were obtained from videotapes during formal testing sessions at 5 and/or 8 weeks after the operation.

Treadmill. A treadmill was used to test both bipedal and quadrupedal locomotion. Reflex bipedal locomotion was checked routinely in all animals by supporting the forelimbs while the hindlimbs stepped freely on the treadmill surface. Animals were trained to maintain their heads at the drinking spout on a variable ratio schedule of reinforcement at three speeds $(2,10$, and $20 \mathrm{~cm} / \mathrm{sec})$; a $10 \%$ sucrose solution was dispensed through the spout for every $1-10$ steps taken for a total of $15 \mathrm{~min} / \mathrm{session}$. These training sessions took place $4 \mathrm{~d}$ per week.

Characteristics of step cycles, weight support, limb position, and interand intralimb coordination were evaluated from videotapes. A step cycle was counted as a movement of a limb that included ankle extension and flexion. Weight support of hindquarters was considered to have occurred in steps in which the hindquarters were seen to be raised above the surface of the treadmill. A weight-supported step included stance and swing phases with hindquarter support. Limb position during stepping was evaluated for external rotation and hyperextension. Dorsal stepping, in which the dorsum of the foot contacted the surface, was distinguished from normal plantar stepping.

Wide and narrow runways. Conditioned overground locomotion was studied on a wide and a narrow runway. Animals were trained to cross in both directions to the sound of a bell for a sucrose solution reward for a total of four crossings/day, three times/week. The wide runway consisted of clear Plexiglas (165 cm long, $23 \mathrm{~cm}$ wide, $122 \mathrm{~cm}$ high) with masking tape applied to the surface to improve traction; the narrow runway had the same dimensions except that it was $5 \mathrm{~cm}$ wide. A safety net was attached under the runways to prevent injury if animals fell from the runways. 
Videotapes taken from the lateral aspect were analyzed for hindquarter weight support and interlimb intervals (interval between stance and liftoff of homolateral limbs) and rates of stepping. Videotapes of ventral views were used to obtain measurements of foot placement relative to body axis and footfall patterns.

Staircase descent. The pattern of footfall and weight support was recorded in animals trained to descend stairs. Animals were trained to descend to the sound of a bell to obtain a water reward at the bottom of the staircase. For weanlings up to $100 \mathrm{gm}$, stairs were $1.3 \mathrm{~cm}$ high, $3.2 \mathrm{~cm}$ deep, and $9 \mathrm{~cm}$ wide. In older animals, the individual stairs were $2.5 \mathrm{~cm}$ high and $3.8 \mathrm{~cm}$ deep. Sessions usually consisted of five trials, three times/week. Weight support and footfall pattern were measured from videotapes of the lateral aspect.

Horizontal ladder crossing. Animals were trained to cross a horizontal ladder in both directions for a water reward to the sound of a bell for a total of four crossings/day, three times/week. A transparent plexiglass ladder was used, with $1.3-\mathrm{cm}$-diameter round rungs, spaced $5 \mathrm{~cm}$ apart, raised $2 \mathrm{~cm}$ above the runway surface by horizontal supports. The runway was $165 \mathrm{~cm}$ long and $23 \mathrm{~cm}$ wide. Crossings were filmed from both lateral and ventral views. Foot placements on rungs with hindquarter weight support were recorded. Placement of the foot between rungs rather than on a rung or foot placement on a rung without hindquarter weight support were counted as failures.

Retransection surgery. To assess the function that was dependent on axons that descend into or through the transplant from the brain or rostral spinal cord, four animals that had been trained on the treadmill were subjected to retransection surgery as adults. Two trained rats that had received transplants as neonates and two trained rats spinalized as neonates had their spinal cords retransected at T5-6, just rostral to the site of the original lesion. The animals were anesthetized, and their spinal cords were exposed rostral to the original laminectomy site and transected using iridectomy scissors. After surgery, animals were warmed gently under a heating lamp and returned to their home cages. Bladders were expressed every $10 \mathrm{hr}$ after the operation until there was evidence of development of reflex bladder function, i.e., bedding was damp and the bladder was empty after expression. Antibiotic injections were given daily, beginning immediately after surgery and continuing for a period of up to 2 weeks. The rats were observed for 5 weeks for withdrawal reflexes to painful and nonpainful stimuli, spontaneous reflexes, and frequency of weight-supported steps on the treadmill. Controls were two trained rats that were transected as adults.

Statistics. The distribution of scores for transplant and spinal animals was bimodal. Therefore a Mann-Whitney analysis was used to test significance of differences in percentage of weight-supported steps between transplant and spinal animals. Interobserver reliability for these measures was high $(r=0.96)$.

\section{Anatomical analysis}

Animals were killed after the completion of behavioral testing. Some animals were used for additional studies; as a result, survivals ranged from 6 weeks to 8 months. Animals were anesthetized deeply and perfused intracardially with $0.9 \%$ physiological saline followed by $4 \%$ paraformaldehyde with $0.3 \%$ picric acid fixative in $0.1 \mathrm{M}$ phosphate buffer. The spinal cord was dissected out, and blocks were prepared for cryostat sectioning. Blocks rostral and caudal to the area of the neonatal lesion were cut in serial transverse $20 \mu$ sections; blocks containing the lesion/ transplant were cut in serial, sagittal $20 \mu$ sections. In all animals, adjacent spinal cord sections through the lesion site were stained with a Nissl/myelin stain to verify the lesion and assess the morphological characteristics of the transplant. Antibodies to serotonin (5HT) were used as a marker for descending axons that had grown into or through the transplant. In some animals, additional sections were stained with antibodies to microtubule-associated protein-2 (MAP2) to identify neurons within the transplant, with antibodies to calcitonin gene-related protein (CGRP) to identify dorsal root axons that had regenerated into or through the transplant, with antibodies to glial fibrillary-associated protein (GFAP) to stain astrocytes, and with antibodies to OX42 to stain activated monocyte-derived cells. Cross sections rostral and caudal to the lesion sites were stained with a Nissl/myelin stain and with antibodies to $5 \mathrm{HT}$ and in some cases with antibodies to GFAP and CGRP. Commercial antibodies (5HT, Incstar, Stillwater, MN, diluted 1:1000; CGRP, Peninsula, Belmont, CA, diluted 1:16,000; GFAP, Biomedical Technologies, Stoughton, MA, diluted 1:1000) and antibodies to MAP2 (dilution 1:1000), kindly provided by Dr. Itzhak Fischer, were used. Frozen sections were mounted on slides and incubated with the appropriate primary antisera for $24 \mathrm{hr}$ and then with biotinylated goat anti-rabbit $\mathrm{IgG}$ and with avidin-biotinylated horseradish peroxidase complex as specified by the manufacturer (Vectastain ABC Kit, Vector Laboratories, Burlingame, CA). Peroxidase activity was visualized with $0.05 \%$ diaminobenzidine tetrahydrochloride and $0.01 \%$ hydrogen peroxide in $0.05 \mathrm{M}$ Tris buffer. Control sections, prepared using preimmune serum, showed no staining.

All histological preparations were examined by two or more investigators who did not know the motor performance of the animal. The completeness of the transection was assessed by the absence of continuity between rostral and caudal stumps in serial sections through the lesion site. Transplanted tissue in the transection site was identified by cellular tissue that did not show the laminar organization of normal spinal gray matter or organized myelinated tracts. The area of integration between the transplant and the host was demarcated by a region of small cells; these were identified as astrocytes by GFAP staining or as monocytederived cells by morphological criteria or by OX42 staining. The cells within the transplant were identified as neurons by morphological criteria or by positive staining with MAP2 antibodies. Cross sections rostral and caudal to the lesion site were examined with Nissl/myelin staining for structural integrity and for 5HT immunoreactivity.

The distribution of 5HT immunoreactivity in cross sections caudal to the transplant was mapped. Images were acquired in bright field at $20 \times$ using a Leica microscope equipped with a Sensys CCD camera. Using IP lab software, grayscale images through the cross section were collected and arranged as a montage. The montage was then transferred to Adobe Photoshop software and a two-layer image was created, the first layer corresponding to the montage and the second corresponding to a transparent sheet over the montage. At high magnification, the 5HT immunoreactive fibers and the outline of the gray and white matter were drawn on the second layer. The second layer image was then printed, producing the pattern of $5 \mathrm{HT}$ immunoreactive axons within the section.

\section{RESULTS}

The operated animals seemed to be healthy and gained weight, and none required bladder care or other special management. The weights of operated animals, however, were less than those of their control littermates. At 13 weeks, control animals weighed $317 \pm 76 \mathrm{gm}$, transplant rats $216 \pm 70 \mathrm{gm}$, and spinal rats $164 \pm$ $16 \mathrm{gm}$. Control animals were thus significantly heavier than spinal animals (Tukey, $p<0.05$ ), but weights of spinal and transplant animals did not differ significantly from one another.

\section{Reflex locomotion}

In bipedal treadmill locomotion, the investigator supports the animal's torso and forelimbs, and the hindfeet are in contact with the moving treadmill surface and show alternating stepping movements. This tests the integrity of the lumbar spinal pattern generator; good reflex locomotion was predicted because the pattern generator was at least partially intact in all three groups. The only animal in the entire series in which reflex locomotion could not be elicited was a spinal animal whose caudal spinal cord proved to be infarcted and contained no recognizable neurons (data from this animal were not included in the results). At 3 weeks of age and throughout the period of testing, other transplanted and spinal rats performed similarly to control animals during bipedal treadmill locomotion. All three groups of animals showed reflex hindlimb step cycles in response to the movement of the treadmill surface. Most animals were able to vary their speed of stepping according to the treadmill speed over a range from $2 \mathrm{~cm} / \mathrm{sec}$ to 10 $\mathrm{cm} / \mathrm{sec}$. These animals demonstrated hindquarter weight support, often with plantigrade foot placement onto the surface. Control animals never showed dorsal stepping at any speed; dorsal stepping sometimes occurred in both spinal and transplanted rats at the higher speeds.

\section{Conditioned locomotion}

The tests that we used were readily learned and executed by all control rats. The three groups showed qualitative and quantitative 

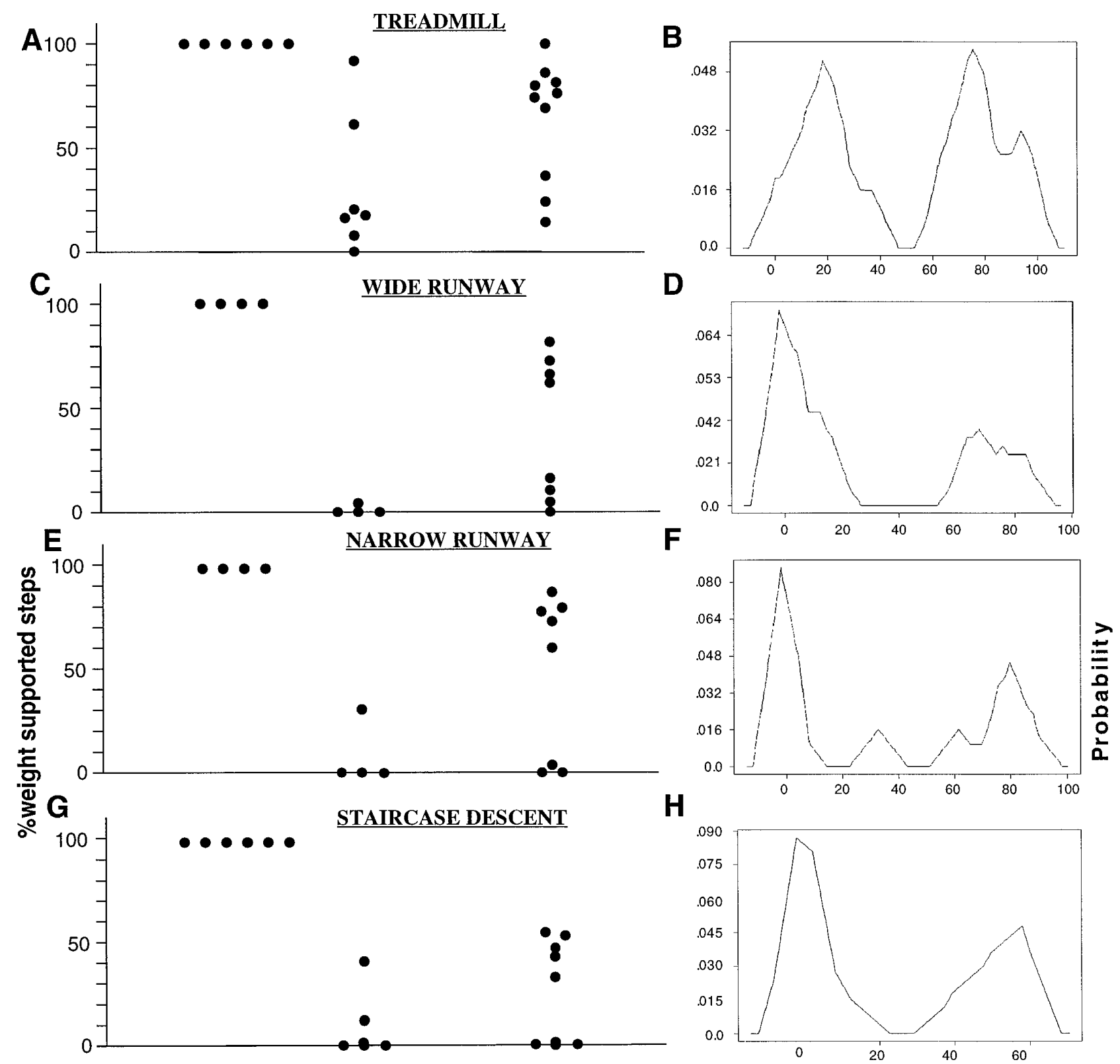

H
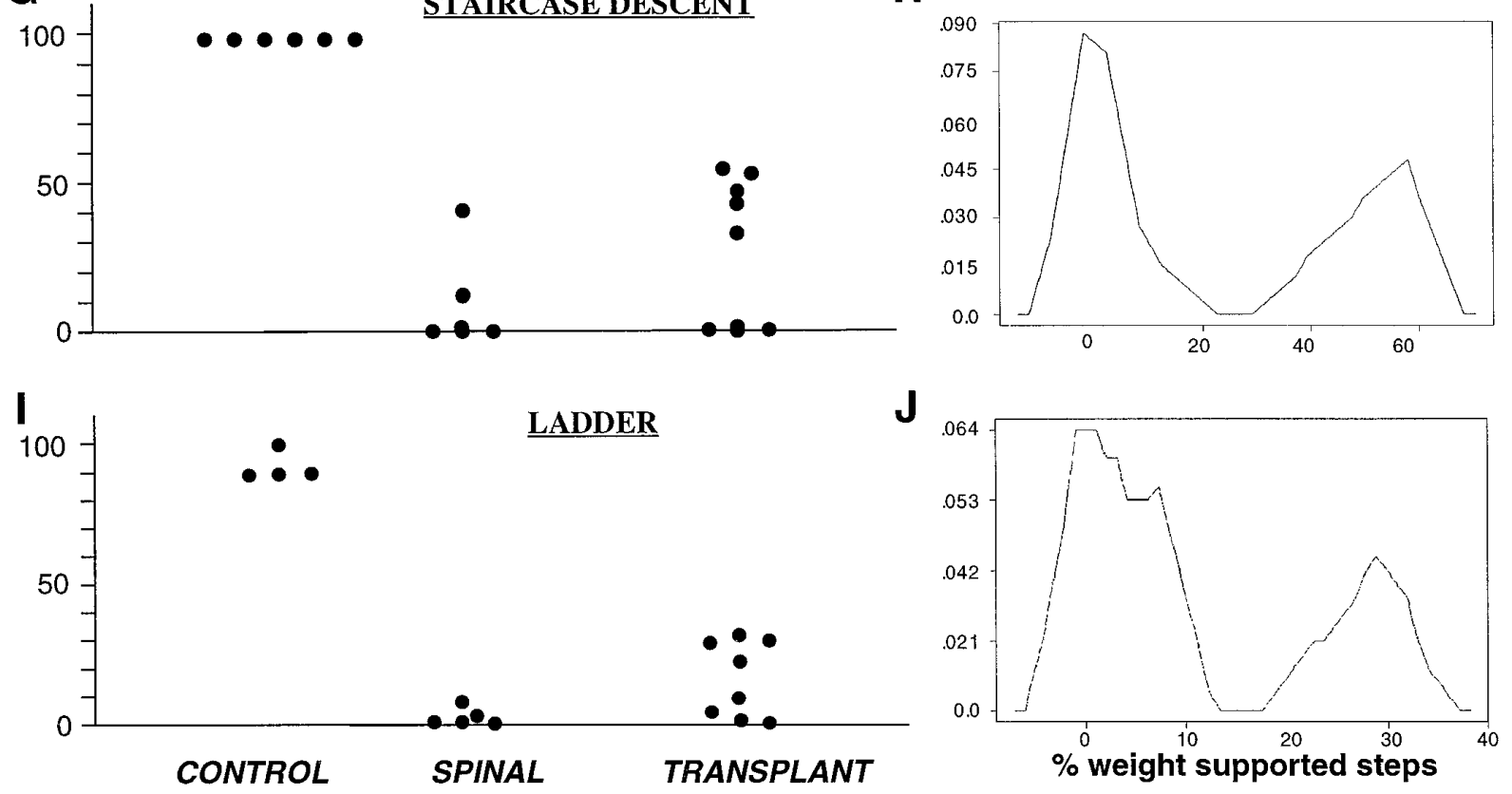

Figure 1. Percentage of weight-supported hindlimb steps used in $(A)$ treadmill locomotion, traversal of $(C)$ wide and $(E)$ narrow runways, $(G)$ stair descent, and $(I)$ horizontal ladder crossing. Each point represents the percentage of weight-supported steps by one control, spinal, or transplant animal recorded during the test period. For each test, the control animals are superior to the operated animals and the transplant group is superior to the spinal group (Mann-Whitney; $p<0.5$; one-tailed). Spinal and transplant animals that performed poorly on the treadmill also performed poorly on the other tests. $B, D, F, H$, and $J$ show the probability distribution of the population's percentage of weight-supported steps for operated (spinal + transplant) animals on the five locomotor tests. The probabilities were generated using a linear kernel smoothing of the histogram data. The bimodal distributions indicate that operated animals can be placed into two performance classes: weight-supported and nonweight-supported. The weight-supported class contains significantly more transplant than spinal animals in all tests. 

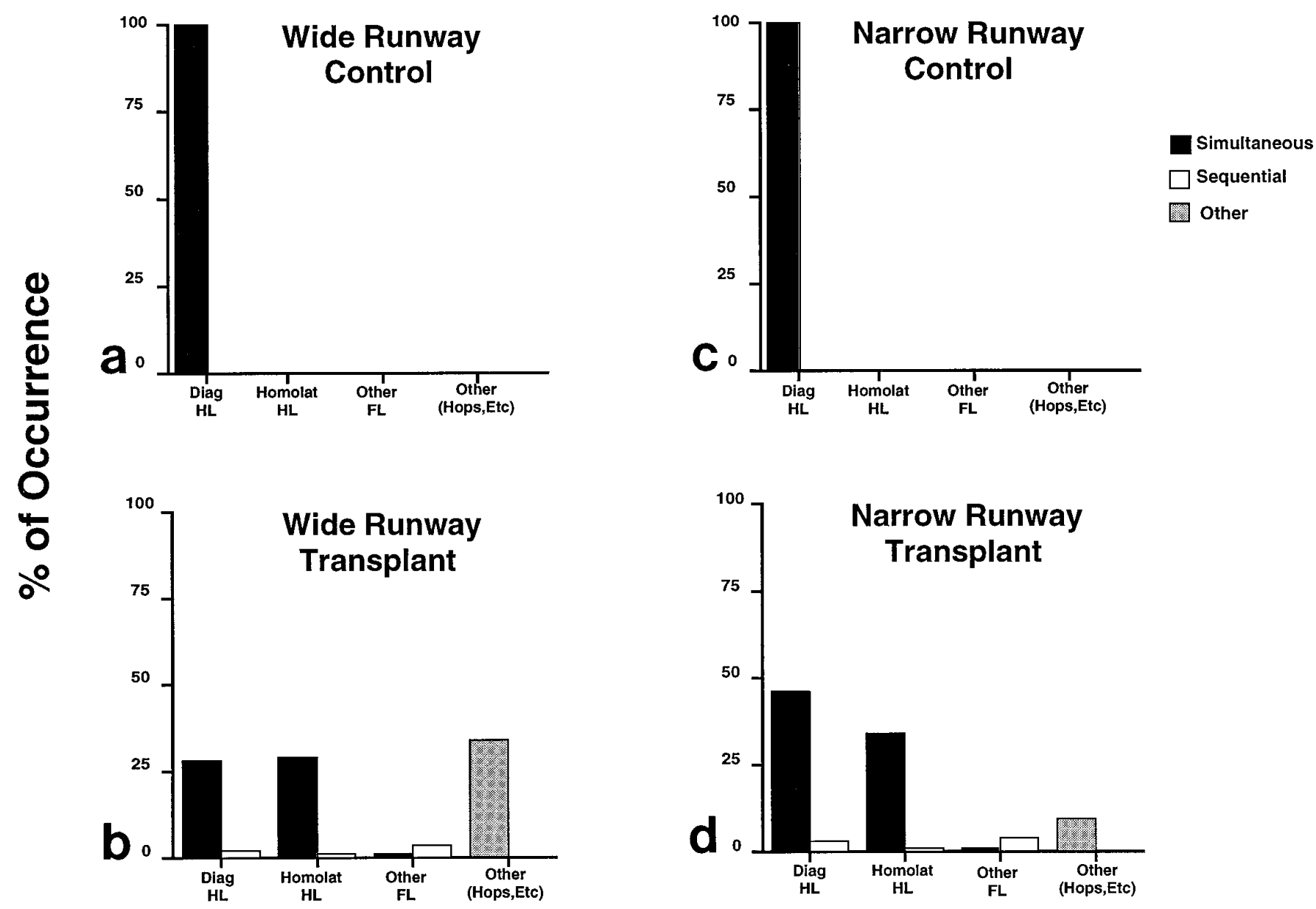

\section{Other Limbs Placed Simultaneously or Sequentially with Forelimb Placement}

Figure 2. Footfall patterns used by normal $(a, c)$ and transplant $(b, d)$ rats on wide $(a, b)$ and narrow $(c, d)$ runways. Normal rats always place forefoot and diagonal hindfoot simultaneously while crossing both wide and narrow runways. Transplant rats use several different patterns on wide and narrow runways. On narrow runways $(d)$, transplant rats decrease the number of unclassifiable hindlimb movements and increase the number of simultaneous or closely sequential diagonal footfalls.

differences on each of the tests. The percentages of weightsupported hindlimb steps on treadmill, runways, stair descent, and horizontal ladder for animals in each group are shown in Figure 1.

\section{Quadrupedal treadmill locomotion}

In quadrupedal treadmill locomotion, the bodies of the rats are not supported by the investigator. To receive the water reward, the animal must locomote on the moving treadmill at an appropriate velocity to keep its body at and its head aligned with the water spout; this can be achieved by using the forelimbs only or by using both fore- and hindlimbs. Differences among groups became apparent in tests of quadrupedal treadmill locomotion. Control animals demonstrated $100 \%$ weight-supported steps with the limbs and feet positioned under the body (Fig. $1 A$ ). The fore- and hindlimbs used an alternating diagonal pattern of coupled coordination while the animal was locomoting. All control animals used plantigrade stepping patterns in all steps.

No animals within the two operated groups showed control levels of hindlimb weight-supported stepping on the treadmill (Fig. 1A). Within both the spinal and transplant groups, there was considerable variability among animals, with some in each group showing high frequency of weight-supported steps and some in each group showing almost no weight-supported hindlimb stepping. A probability distribution (Fig. $1 B$ ) indicates that the performance of operated animals can be divided into two classes: weight-supported $(>60 \%$ weight-supported steps) and nonweight-supported ( $<60 \%$ weightsupported steps). Transplant animals were significantly more highly represented in the weight-supported group than were the spinal animals (Mann-Whitney; $p<0.05$; one-tailed).

Five of the seven spinal animals showed poor hindquarter weight support on most steps, so that the hindquarters and torso were usually in contact with the surface of the treadmill. The hindfeet and hindlimbs were rarely positioned beneath the hindquarters. As these spinal animals matured (during the second and third month), hindquarter weight support decreased further and limbs were usually dragged lateral to the body. One animal (TX13) showed moderate weight support. One spinal animal, TX10, differed from the others in this series. This rat showed good weight support on all tasks, with $89 \%$ weight-supported steps on 

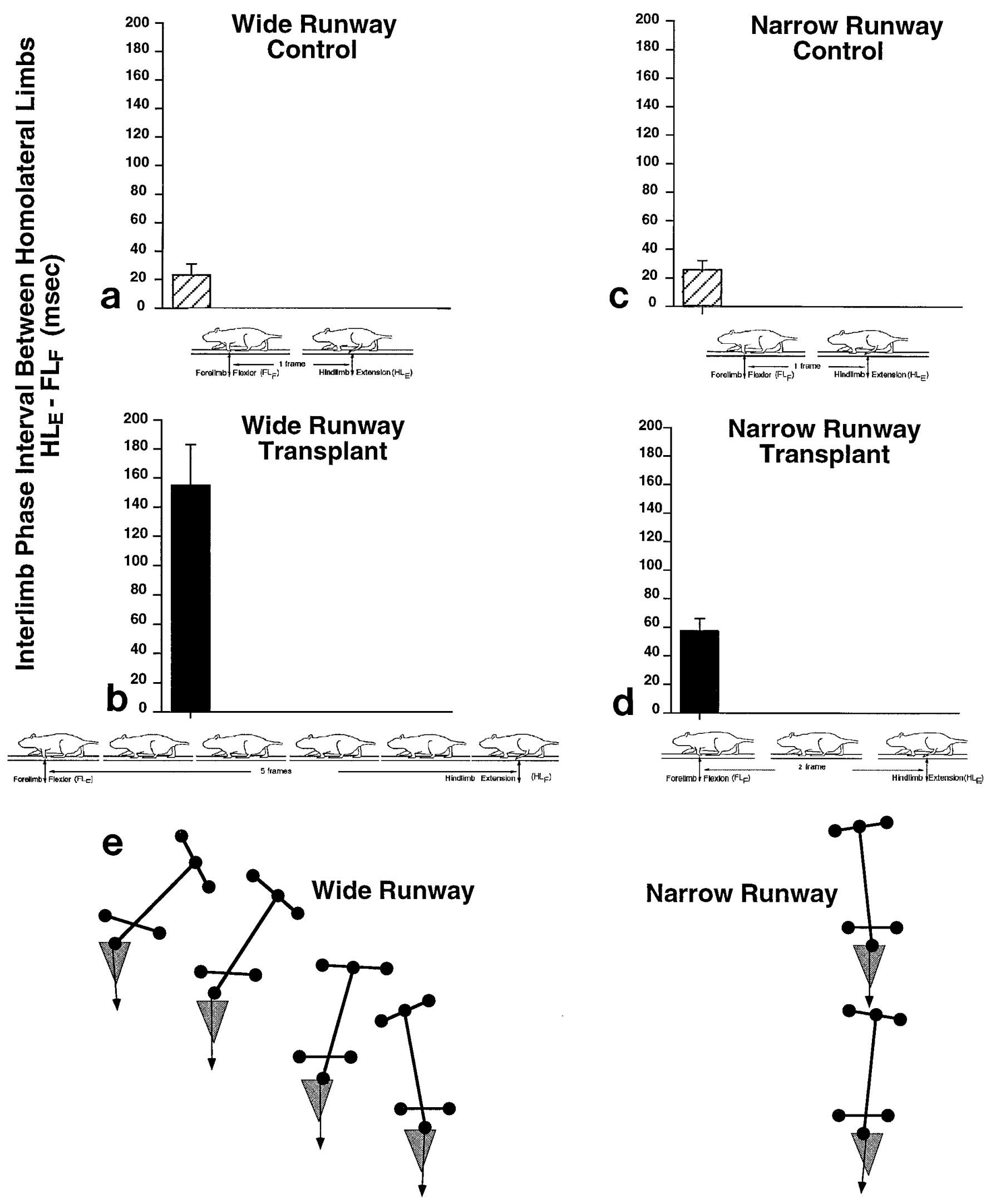

Figure 3. Interphase intervals between homolateral hindlimb extension and forelimb flexion in control $(a, b)$ and transplant $(c, d)$ rats on wide $(a, c)$ and narrow $(b, d)$ runways. The interphase interval is small (within two frames) and similar for control animals on both wide and narrow runways. The intervals are large for transplant animals on the wide runway $(c$, six frames) but reduced toward normal levels $(d$, three frames) on the narrow runway. $e$, Diagram of meandering path taken by a typical transplant animal on the wide runways and the straight path taken on the narrow runway. 
Table 3. Conditioned overground locomotion (wide runway)

\begin{tabular}{|c|c|c|c|c|c|c|c|}
\hline & $n$ & $\begin{array}{l}\text { Mean body } \\
\text { axis relative } \\
\text { to runway } \\
\text { (degrees) }\end{array}$ & $\begin{array}{l}\text { Rotation of } \\
\text { left hindfoot } \\
\text { relative to } \\
\text { body (degrees) }\end{array}$ & $\begin{array}{l}\text { Rotation of } \\
\text { right hindfoot } \\
\text { relative to body } \\
\text { (degrees) }\end{array}$ & $\begin{array}{l}\text { Interlimb } \\
\text { interval } \\
\text { (msec) }\end{array}$ & $\begin{array}{l}\text { Stride length } \\
(\mathrm{cm})\end{array}$ & $\begin{array}{l}\text { \% Weight- } \\
\text { supported } \\
\text { steps }\end{array}$ \\
\hline Control & 4 & $7.21 \pm 1.3$ & $13.1 \pm 2.6$ & $16.9 \pm 12.3$ & $<30$ & 7.1 & 100 \\
\hline Transplant & 6 & $20.0 \pm 5.3$ & $40.31 \pm 8.3$ & $48.0 \pm 10.6$ & $154.1 \pm 28.3$ & 6 & 53 \\
\hline
\end{tabular}

the treadmill at 8 weeks of age. Hindquarter elevation decreased compared with normal or transplant rats as this animal matured; the step cycles were not fluid and the range of motion of the hindlimb joints was diminished. Dorsal stepping was seen in some of the weight-supported steps made by this and the other spinal animals.

Transplanted rats also showed variability in weight-supported locomotion on the treadmill. Of the 10 transplant rats whose behavior was quantified, seven animals (TP14, -17, -18, -19, -20, $-24,-26)$ demonstrated $67-98 \%$ hindlimb weight-supported steps with smoothly executed step cycles throughout the period of training. Three animals (TP21, -23, -26) used $<40 \%$ weightsupported steps; their step cycles were often interrupted or irregular and their limbs were frequently dragged lateral to the body. Some transplant rats occasionally demonstrated dorsal stepping (mean 15\%; range, $0-56 \%$ ). Some of the hindlimb steps by transplant rats were hypermetric, with exaggerated hip flexion. Detailed kinematic analyses of control, spinal, and transplant rats during treadmill locomotion will be described elsewhere (C. Smeraski and S. Giszter, unpublished observations).

\section{Conditioned overground locomotion}

Wide runway. Animals from all three groups were able to cross the wide runway. The control animals used a 1:1 coupling of diagonal hindlimb-to-forelimb gait pattern. All steps were weightsupported (Fig. 1C). The pattern of locomotion was uniform; contralateral pairs of forelimb and hindlimb were engaged in approximately the same portion of the step cycle at the same time (Fig. 2). Details of the step cycle were therefore consistent (Table 3). The interlimb interval, i.e., the time interval between the onsets of stance (E) in the forelimb and swing (F) in the homolateral hindlimb $\left(\mathrm{HL}_{\mathrm{E}}-\mathrm{FL}_{\mathrm{F}}\right)$, was thus constant and short, often less than one frame $(<30 \mathrm{msec})$, in the control rat (Fig. 3). This observation demonstrates the high degree of forelimb/hindlimb coordination in control rats. Dorsal stepping was never exhibited by normal rats during overground locomotion. Also, errors of foot placement were never seen. Stepping was uniform and smoothly executed, with an average stride length of $7.1 \mathrm{~cm}$. Measurements from ventral views showed that the axes of the head and body were in register with the direction of forward movement (Table 3).

Spinal animals traversed the wide runway without hesitation, using their forelimbs. Three of the four spinal animals used few or no weight-supported hindlimb steps (Fig. 1C); therefore, measurements of limb and body orientation were not made for this group. One spinal animal (TX10) used 91\% weight-supported hindlimb steps during early training ( 5 weeks). By 8 weeks, however, weight-supported steps by this animal had decreased to $6 \%$, and locomotion was increasingly achieved by forelimb steps, with the hindlimbs dragged passively. All spinal animals demonstrated some nonweight-supporting hindlimb step cycles in which the hindlimbs moved in a very rapid scissoring motion. While locomoting, the animals maintained a relatively straight body axis $\left(13.3 \pm 5.2^{\circ}\right)$ with respect to the direction of forward movement on the wide runway, and as a result did not fall from the runway.

Animals with transplants were able to cross the wide runway successfully using quadrupedal locomotion, but their locomotor impairment was more obvious than on the treadmill. Weightsupported steps were used, but there was considerable variability (range, 0-84\%), with four animals (TP16, -17, -18, -20) using $>60 \%$ and four (TP19, -21, -23, -24) using $<20 \%$ weightsupported steps (Fig. 1C). Stepping was not uniform and could include dragging the feet on the plantar surface, hopping, or making additional steps with the hindlimbs. Table 3 compares the characteristics of body and limb orientation and stepping for the subset of transplant animals that made $>10 \%$ weight-supported steps with those of control animals. Hindlimb steps made by transplant rats were occasionally hypermetric, with exaggerated hindlimb abduction and rotation of the hindfoot relative to the body. The stepping pattern was less constant in transplanted than in control rats, in part because of the increased mean interlimb interval $(154.7 \pm 28.3 \mathrm{msec})$, fivefold greater than in control animals. In addition, forelimb stance was paired with homolateral hindlimb stance, as is characteristic of the normal rat, in only $27 \%$ of the steps, and approximately one third of the steps were unclassifiable hops or shuffles. Dorsal stepping was common, occurring in $21 \%$ of step cycles. The hindquarters were unstable in all animals; there was considerable lateral roll and yaw of the hindquarters, which produced poorly controlled oscillations of the hindquarters with respect to the head and the direction of forward movement. Although the pronounced roll and yaw indicated impaired postural stability, it rarely resulted in falls onto the runway and never resulted in falls from the runway. As a consequence, however, the locomotor path taken by the animal meandered and included the entire width of the runway, indicating impaired but intermittent control of hindlimb placement and stepping.

The frequency of weight-supported steps by all operated rats again showed a bimodal probability distribution (Fig. 1D), but in this test the weight-supported group ( $>50 \%$ weight-supported steps) was composed only of transplant animals.

Narrow runway. Because the narrow runway was approximately the width of the rat, alignment of hindquarters along the axis of the runway was required to permit effective traversal and to prevent falls. Control animals locomoted across the narrow runway as quickly and almost as precisely as on the wide runway (Fig. $1 E$, Table 4). All steps were weight supporting, and animals used the typical 1:1 coupling of alternating forelimb to diagonal hindlimb foot placement (Fig. 2). The mean interlimb interval on the narrow runway, as on the wide runway, was $<30 \mathrm{msec}$ (Fig. 3). The average stride length was similar to that on the wide runway $(6.6 \mathrm{~cm})$. Ventral views of control rats showed that the alignment of body axis $\left(3.3 \pm 1.3^{\circ}\right)$ and of right hindfoot $\left(12.6 \pm 2.1^{\circ}\right)$ and left hindfoot $\left(9.5 \pm 2.4^{\circ}\right)$ was nearly parallel with the runway and the forward trajectory of movement (Table 4). Dorsal stepping 
Table 4. Conditioned overground locomotion (narrow runway)

\begin{tabular}{|c|c|c|c|c|c|c|c|}
\hline & $n$ & $\begin{array}{l}\text { Mean body } \\
\text { axis relative } \\
\text { to runway } \\
\text { (degrees) }\end{array}$ & $\begin{array}{l}\text { Rotation of } \\
\text { left hindfoot } \\
\text { relative to } \\
\text { body (degrees) }\end{array}$ & $\begin{array}{l}\text { Rotation of } \\
\text { right hindfoot } \\
\text { relative to body } \\
\text { (degrees) }\end{array}$ & $\begin{array}{l}\text { Interlimb } \\
\text { interval } \\
\text { (msec) }\end{array}$ & $\begin{array}{l}\text { Stride length } \\
(\mathrm{cm})\end{array}$ & $\begin{array}{l}\text { \% Weight- } \\
\text { supported } \\
\text { steps }\end{array}$ \\
\hline Control & 4 & $3.3 \pm 1.3$ & $9.5 \pm 1.4$ & $12.6 \pm 2.1$ & $<30$ & 6.6 & 100 \\
\hline Transplant & 6 & $10.6 \pm 2.3$ & $24.6 \pm 5.8$ & $23.7 \pm 6.3$ & $56.7 \pm 9.2$ & 6.9 & 65 \\
\hline
\end{tabular}

and other errors of foot placement were never seen on the narrow runway.

The locomotor behavior on the narrow runway of three of the four spinal animals was also similar to that used on the wide runway, because forward movement was achieved by the forelimbs. These spinal rats never made hindlimb weight-supported steps while traversing the narrow runway (Fig. $1 E$ ). The number of hindlimb step cycles, however, was increased, but as a result of increased scissoring rather than weight-supported steps. Their inability to make compensatory postural adjustments of the hindquarters often resulted in falls from the runway into the safety net. Some spinal animals, particularly as they became more experienced, would occasionally "freeze" for up to 2 min rather than cross the narrow runway, a behavioral response that was not seen in control or transplant rats. One spinal rat, TX10, used $70 \%$ weight-supported steps on the narrow runway at 3 weeks and $33 \%$ at 8 weeks and thus again distinguished herself from the other spinal rats; she also showed a deterioration with age.

Locomotion across the narrow runway by transplant rats was less accurate, more variable, and slower than for control rats. Surprisingly, those transplant rats that showed good weightsupported locomotion on the wide runway improved in all measures of inter- and intralimb coordination on the narrow runway, indicating a change in locomotor strategy for the narrow runway (Figs. 1E, 2, 3; Table 4). Of the right forelimb steps, $48 \%$ were coupled to the alternate left hindlimb step, and homolateral forelimb/hindlimb couplings occurred in 33\% of the steps; unclassifiable support patterns, including hops and shuffles, decreased to $<20 \%$ (Fig. 2). The mean interlimb interval was $56.7 \pm 9.2 \mathrm{msec}$, approximately one third of that on the wide runway (Fig. 3). The average stride length was similar to that seen in controls: more of their hindlimb steps were weight supporting, exaggerated hindlimb flexion was rarely seen, their body axis was maintained almost parallel to the runway, and their hindfeet were placed under the body and in the direction of forward movement (Table 4). Because hindlimb abduction was rarely seen on the narrow runway, the transplanted animal was able to use a step-support pattern strategy similar to, although not identical to, that of control animals. The lateral instability of the hindquarters that resulted in pronounced roll and yaw on the wide runway was virtually abolished, permitting the transplant rats to traverse successfully the more challenging runway. Hindlimb slips off the runway did occur, but in $20 \%$ of the cases postural corrections were made quickly, and neither falls from the runway nor freezing were seen. Dorsal stepping was rare $(7.7 \%)$ on the narrow runway. These data indicate much closer coupling of coordination between fore- and hindlimbs with respect to timing and footfall patterns induced by the challenge of crossing the narrow runway.

The frequency of weight-supported steps on the narrow runway by operated animals was bimodal (Fig. $1 F$ ), and all animals with good locomotion ( $>50 \%$ weight-supported steps) were those with transplants.
Staircase descent. Staircase descent requires appropriate adjustment of postural support for the hindquarters coordinated with a pattern of forward propulsion and braking by the limbs. Control rats descended stairs using $100 \%$ weight-supported steps (Fig. 1G, 4 ) in a characteristic pattern in which diagonal limbs were moved simultaneously and placed on alternate stairs so that two limbs were never supported by the same stair at the same time. The feet were placed at the forward edge of the stair with the toes curled over the edge. Very young control pups adopted a three-legged support pattern in which each limb moved sequentially to the next step so that both forelimbs or both hindlimbs could be supported on the same step; by the age at which formal training began, however, control rats used the adult pattern of stair descent.

The additional challenge in staircase task for operated animals is to maintain accurate control of the hindquarters during descent. Most spinal rats demonstrated few or no hindlimb weightsupported steps (Fig. $1 G$ ) during successful descents (those that did not resulted in falls). Forelimbs were used to descend one stair at a time, and the hindlimbs were dragged from step to step. The best performing spinal rat, TX10, differed in that many steps were weight-supported, although the hindquarters were unstable and foot placement was variable and poorly controlled. Successful descent by this rat was accomplished through various patterns of foot placement, including hops, two- and three-leg support patterns, and bracing the hindfeet against the riser, interspersed with dragging of hindlimbs.

Transplant rats also showed hindquarter instability and falls during descent. Some of the transplant rats that performed well on treadmill and runways (TP16, -18, -19, -21) were able to use hindlimb weight-supported steps to descend stairs (Fig. 1G). In these steps, the plantar surface of the foot was placed in the middle or front of the stair, and the hindquarters were elevated. During the early stages of training, the transplant rats with weightsupported stepping consistently used a three-legged support pattern of staircase descent, a strategy resembling that used by young control pups. Each forelimb was moved sequentially to the next step, followed by the hindlimbs. Thus, either both forelimbs or both hindlimbs were supported on the same stair simultaneously. This pattern of braking and restarting proceeded slowly, with the animals maintaining a hunched trunk posture throughout descent. During later stages of training, three (TP16, -18, -19) of the four transplant rats that showed good weight-supported steps developed a two-legged support pattern, similar to that used by adult control animals, and most of these steps were weight-supported.

A bimodal distribution of weight-supported steps by operated animals was also seen in staircase descent (Fig. $1 H$ ). In this more difficult test, the group showing good locomotion indicated by $>30 \%$ weight-supported steps was composed primarily of transplant animals.

Ladder crossing. In the ladder-crossing task, rats could support their weight using foot placements on the rungs or on the surface approximately $2 \mathrm{~cm}$ below the rungs. We defined successful hor- 
izontal ladder crossing on the basis of the behavior of our control animals. Trained control rats crossed the ladder quickly and efficiently using a 1:1 coupling pattern of diagonal fore- and hindlimbs. This is the same gait pattern used on all other tests of overground locomotion. The ipsilateral hindlimb was placed on every other rung in coordination with the opposite forelimb and with the placement of the hindfoot very near the position occupied by the ipsilateral forefoot. The dorsal surface of the hindfoot never contacted the rung, in either swing or stance phases.

The particular challenge for weight-supported stepping in ladder crossing is the requirement that feet be positioned correctly on the rungs during locomotion and/or that errors in foot placement be corrected. This targeting was achieved with weightsupported steps by all control rats in $\sim 90 \%$ of the steps (Fig. 1I), those steps not placed on the rungs were almost invariably the initial step in a series. Most spinal rats never showed hindlimb weight support on this test. Limbs were usually dragged passively lateral to the body, whereas all locomotion was achieved by the forelimbs targeting rungs and pulling the caudal portion of the body over the ladder. The best performing spinal rat, TX10, showed some weight-supported hindlimb steps, but only $8 \%$ were placed correctly on the rung. Failure to target the rung did not result in repetition of the stepping movement. Hops, in which both hindlimbs moved simultaneously, were the preferred strategy used by this animal. These hops showed minimal clearance above the surface of the ladder.

Transplant rats also locomoted across the ladder with difficulty (Fig. 1I). Forelimbs were moved one at a time in a pattern similar to those of normal rats, but no clear pattern of hindlimb usage was seen. As in normal rats, hindlimbs were usually moved one at a time, but occasionally $(26 \%)$ both hindlimbs were moved simultaneously in a hopping movement. In the best performing transplant animal, only $32 \%$ of the steps were placed on the rungs, and in $18 \%$ of those steps it was the dorsal surface of the foot that initially contacted the rung. Frequently $(51 \%)$ when the hindfoot extended between the rungs, the dorsal surface of the hindfoot brushed the rung during swing, but this did not elicit reflex-placing that corrected the foot position. The poor level of motor precision and impaired ability to coordinate sensory information between the fore- and hindlimbs seem to account for the impaired performance of these animals on the ladder test. Nevertheless, rats with transplants were superior to spinal rats in weight support and controlled use of the hindlimbs to cross the ladder.

This most difficult task also yielded a bimodal distribution of weight-supported steps (Fig. $1 J$ ). All animals with better locomotion ( $>20 \%$ weight-supported steps) had transplants.

\section{Motor behavior after retransection (Fig. 5)}

Trained control animals showed $100 \%$ weight-supported stepping on the treadmill; after transection as adults, they lost this ability, and there was no recovery of weight-supported stepping during the period after the operation. These animals also required manual evacuation of their bladders during the first 2 weeks after operation.

Two animals that were given transplants as neonates and two animals spinalized at birth were retransected as adults at the conclusion of treadmill training. One transplant animal (TP25) had developed good weight-supported locomotion, whereas the second transplant animal (TP26) was an example of a poor performer. The two spinal animals also represented examples of good (TX13) and poor (TX12) treadmill stepping before retransection. Immediately after the retransection, all four animals showed considerable loss of function, but by the end of the first week automatic bladder function and some hindlimb function had recovered. Remarkably, all four retransected rats were able to make some unassisted weight-supported steps, which the two trained rats transected as adults could not do. Thus, after the second transection the neonatally operated animals performed better than did animals transected first as adults.

The poorly performing spinal animal (TX12) showed little change in frequency of weight-supported steps, whereas the better performing animal, TX13, showed a decrease but then recovered to its preretransection level of $64 \%$ weight-supported steps. Neither animal changed hindlimb or hindfoot configuration from the preretransection period. The retransection, therefore, had little effect on the performance of the spinal animals after an initial period of depression.

Animals given transplants as neonates and then retransected as adults showed a substantial decrease in weight-supported steps during the first week and some recovery during the next 4 weeks, but neither recovered to preretransection level of weight support. Neither changed the configuration of hindfoot or hindlimb, and neither rat used the hindlimbs in coordinated overground locomotion, although both could do so before retransection. The retransection therefore diminished the motor function of the transplant animals, even after retraining.

\section{Morphology}

Transections were verified by lack of continuity of neural structures studied in serial sagittal sections. There was disruption of the normal spinal cord structure, lack of continuity in myelinated pathways, and absence of lamination of gray matter.

The rostral and caudal margins showed dense accumulations of GFAP-stained cells, indicating an astrocytic scar (Fig. 6a). The spinal cord gray matter rostral and caudal to the transection site was well preserved. Some ascending pathways (dorsal columns) were greatly diminished or absent in sections rostral to the lesion, and some descending pathways (corticospinal) were diminished caudal to the lesion. 5HT staining was robust rostral to the transection and absent caudally in all cases of spinal transection. CGRP staining of dorsal root axons within lamina I and II and of motor neurons was present both rostral and caudal to the lesion. MAP2-stained neurons were present in gray matter both rostral and caudal to the lesion. The transections showed similar morphology among all spinal animals. Specifically, the transection in spinal animal TX10, who performed much better than the other spinal rats, was complete.

Surviving transplant tissue was identified in all of the animals examined. A transplant was considered to have survived if neurons, stained by MAP2 or with the morphology of neurons in Nissl/myelin-stained sections, were recognized in the lesion site. The dorsal columns were much diminished caudal to the lesion/ transplant and the corticospinal tracts diminished rostrally, but the gray matter was well preserved both rostral and caudal to the transplant. A concentration of glial cells identified the border between transplant and host. The size of the transplant and extent of integration varied markedly (Fig. 6b,c). In general, the size of the transplant and the extent of integration was greater in animals killed $<2$ months after operation than in those killed 6-8 months after operation. Transplants rarely completely filled the lesion cavity, and as a result cyst-like spaces were present; these spaces also seemed to be larger in the older animals. A laminar structure resembling normal spinal gray matter was never seen in transplant tissue. Integration with the rostral stump was usually not as 

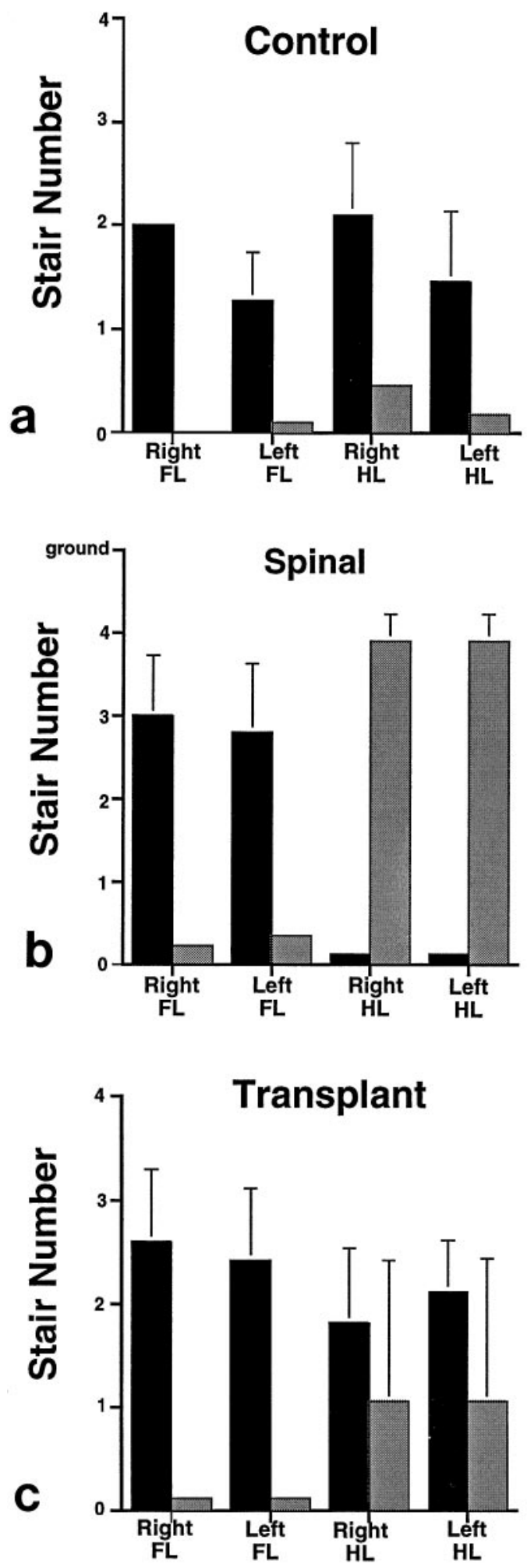

Weight Supported Steps
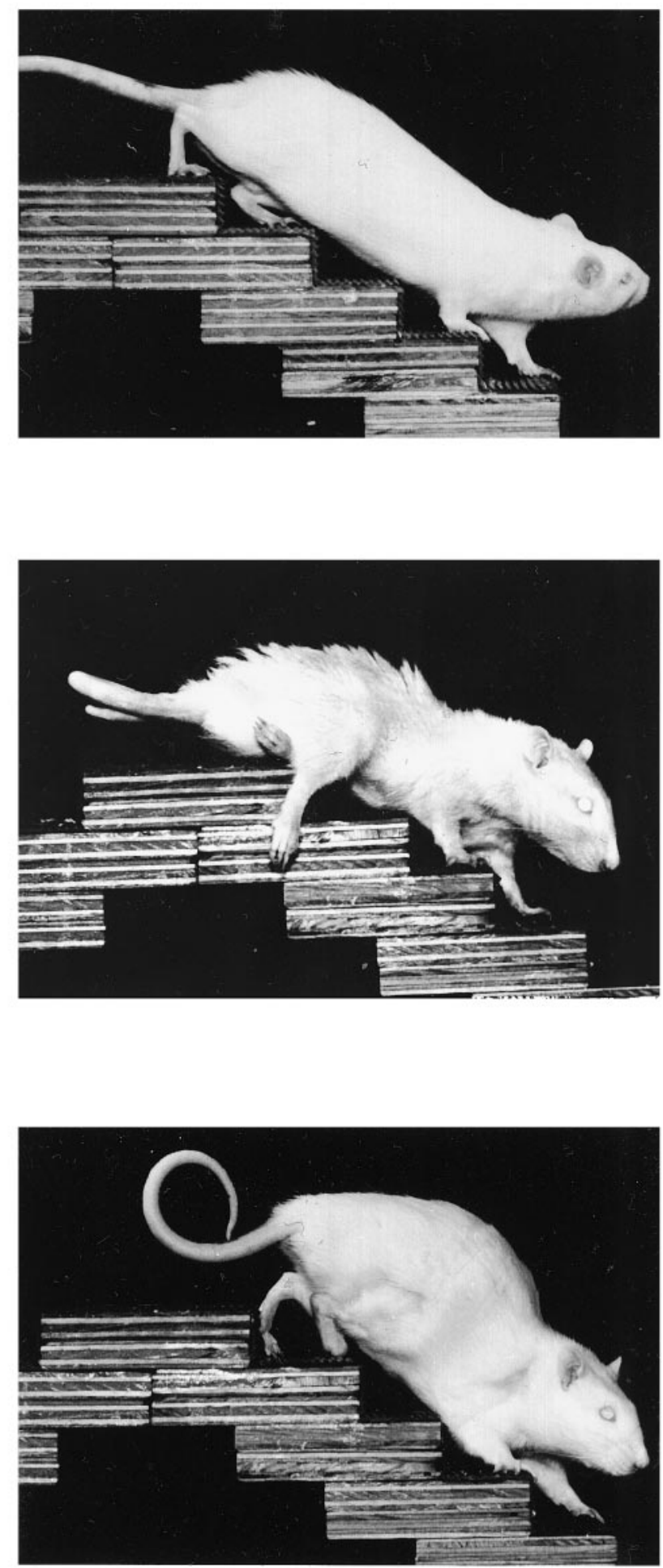

\section{Non-Weight Supported Steps}

Figure 4. Staircase descent by control $(a)$, spinal $(b)$, and transplant $(c)$ rats. Graphs show weight-supported steps taken by each limb on a flight of four stairs. Control rats show virtually all weight-supported steps. Spinal rats use weight-supported forelimb steps but virtually all hindlimb steps are nonweight-supported. Transplant animals use weight-supported forelimb steps and both weight-supported and nonweight-supported hindlimb steps. Photographs of rats descending staircase show good elevation of hindquarters, indicating good weight support, in control and transplant rats. 

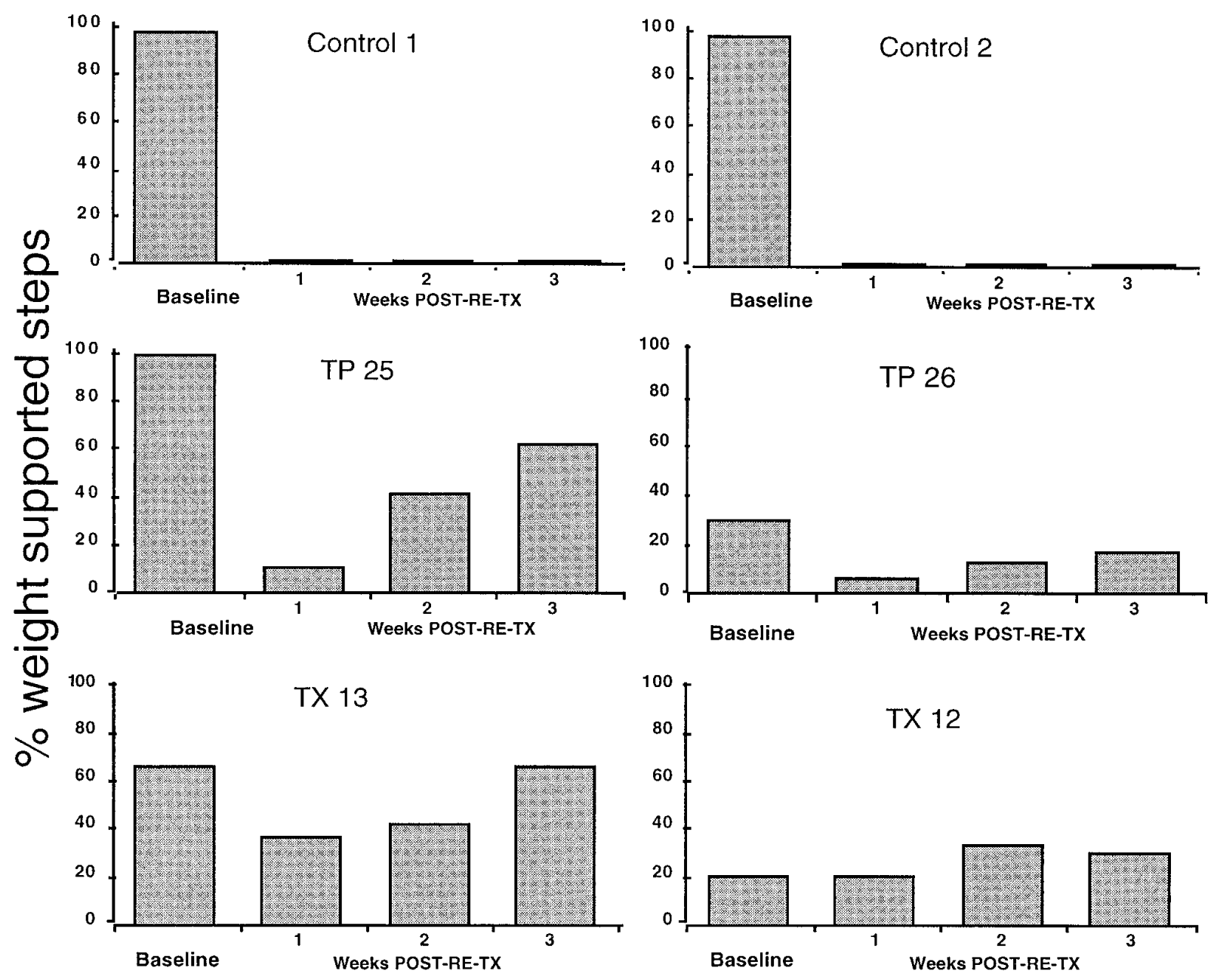

Figure 5. Graphs showing loss and recovery of weight-supported stepping on a treadmill in two trained control animals, transected as adults, and after retransection in two trained adult animals transplanted and two transected as neonates. Control animals permanently lose weight-supported stepping after transection as adults. Both transplant animals recover to $60 \%$ of preretransection level, whereas both spinal rats recover to the level of performance achieved before the second transection.

extensive as with the caudal stump. The transplanted tissue seemed to be extremely fragile, and the connection with the host, particularly in older animals, was tenuous, so that we cannot rule out artifactual separation of areas of integration of transplant from host tissue during processing. Size and integration of the transplanted tissue with neonatal hosts that survived to adulthood was considerably less than that seen when tissue is transplanted into adult hosts with similar post-transplantation survivals or when animals were killed within 1 month of transplantation (unpublished data).

5HT staining indicating the presence of descending serotonergic axons was found caudal to the transplant (Fig. 7) in seven transplant animals. The distribution of 5HT in the transplant animals was quite variable; neither density nor distribution seemed to relate to the motor performance of the animals (compare $7 A-D$, animals that had good treadmill locomotion, with $7 E$, an animal with poor treadmill locomotion). 5HT was not found caudal to the transplant in TP18, which had good (78\% weightsupported steps) locomotion. 5HT was also absent caudal to the transplant in the two animals TP25 and TP26, which received retransections 5 weeks before they were killed; at this posttransection interval, 5HT immunoreactive axons have degenerated (unpublished data). In three of the transplant animals (TP19,
$-20,-21)$, the transplants contained cell bodies of transplanted serotonergic neurons (Fig. 8). The 5HT found caudal to the transplant in these three animals was likely derived at least in part from these transplanted neurons; these transplants were probably taken from high cervical-low medullary regions that contain 5HT neurons. Two of these animals, TP19 and TP20, had good weight support on treadmill locomotion (80 and $88 \%$, respectively), whereas the third, TP21, had only $38 \%$ weight-supported steps on the treadmill. No serotonergic cells were found in the transplants in the other animals, indicating that the 5HT found caudally originated from host neurons.

Dorsal root ganglia were often displaced, so that they abutted the transplant/transection site. Dorsal root axons stained for CGRP were found in some cases to enter the transplant (Fig. 9); this pattern is consistent with relays formed by dorsal root innervation of the transplant (Itoh et al., 1996)

The site of retransection (Fig. 10) in both the spinal and transplant animals indicated a complete lesion, with morphology similar to transections made in neonates. In these animals, however, unlike animals operated on as neonates and unlike animals spinalized and transplanted as adults (unpublished data), OX-42stained cells, indicating activated monocyte derived cells, could be recognized at the lesion site and within the transplant (Fig. 10a). 

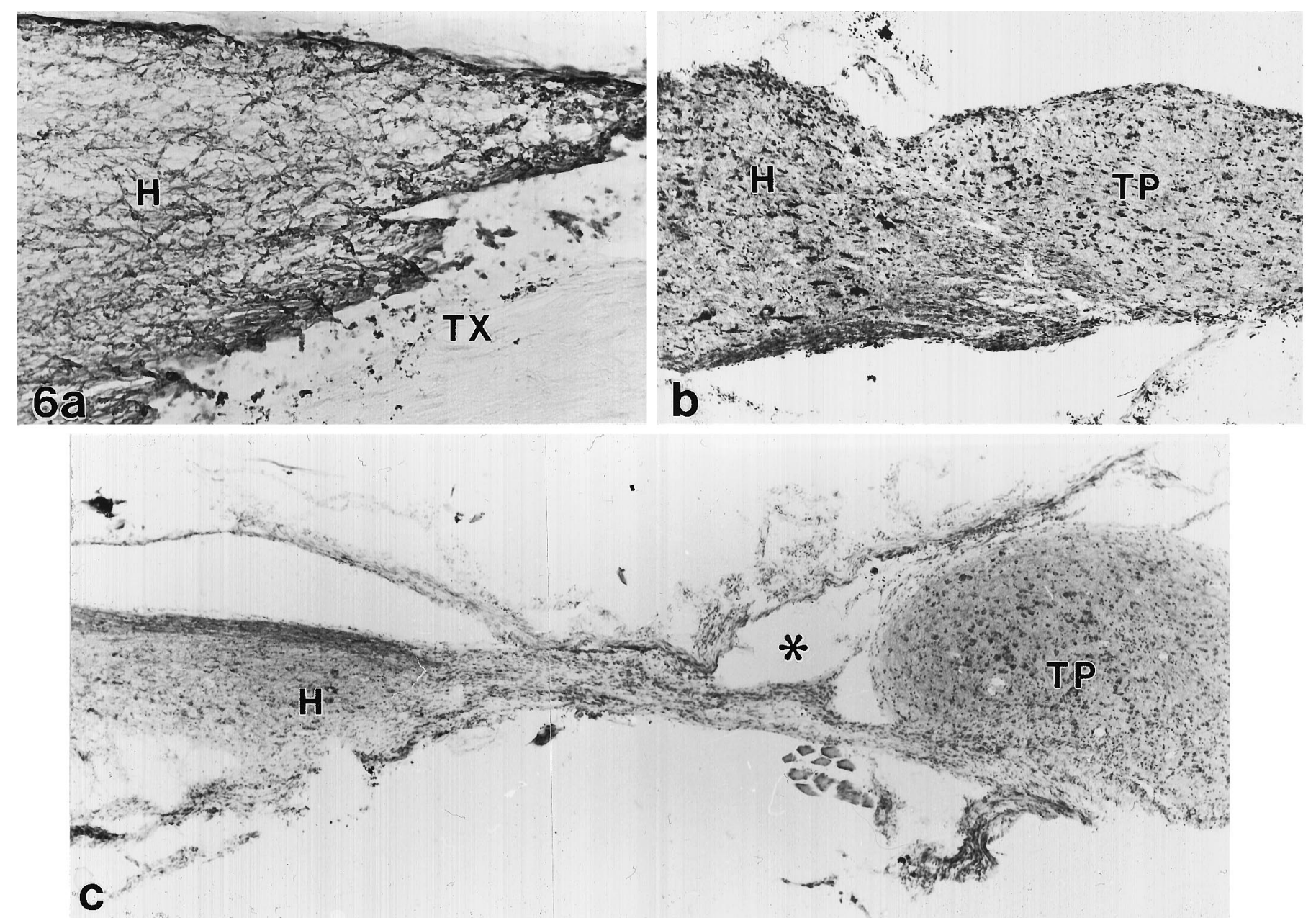

Figure 6. Photomicrographs of transection and transplant sites. $a$, Photomicrograph of transected spinal cord, stained with GFAP immunocytochemistry. Note dense staining indicating concentration of astrocytes at transection $(T X)$ border. $40 \times$ magnification. $b$, Transplant $(T P)$ integrated with host $(H)$ spinal cord; 2 months survival. Cresyl violet stain. $40 \times$ magnification. $c$, Transplant $(T P)$ poorly integrated with host $(H)$ spinal cord. Note cyst (indicated by *); 4 months survival. Cresyl violet stain. $40 \times$ magnification.

The presence of these cells may indicate that a more active immune response was initiated by the second surgery. This immune response seems to affect both retransected spinal and transplant animals. It did not prevent recovery of function in either group of retransected animals and seems unlikely to account for the difference in function between these two groups.

\section{DISCUSSION}

Our analyses of rats that received transplants of fetal spinal cord into the site of a complete transection as neonates demonstrate that some of these animals develop treadmill and overground locomotion that are qualitatively and quantitatively superior to the function achieved by most spinal animals subjected to identical surgical and training protocols, except for the presence of the transplant. Their locomotor responses are sufficiently adaptable to allow these transplant recipients to execute challenging tasks. Of considerable interest is the observation that some of these animals can improve the precision of their locomotion on more difficult tasks.

\section{Normal rats}

During the first few weeks of life, control, spinal, and transplant rats show relatively few qualitative differences, because hindquar- ter support and adaptive postural responses play a minimal role in locomotor function of preweanling pups. The mature motor pattern is based on the rhythmic alternation of flexion and extension in the forelimbs and hindlimbs that is believed to depend on the activity of networks of interneurons that constitute the central pattern generators (CPGs) and are organized within the cervical and lumbar spinal segments; rhythmic alternating patterns are demonstrable in neonates (Kudo et al., 1991; Cazalets et al., 1994; Howland et al., 1995a; Kiehn and Kjaerulff, 1996). In mature animals the activity of cervical and lumbar CPGs is coordinated to give a characteristic pattern of locomotion that we observed to consist of a 1:1 coupling pattern of diagonal forelimbs and hindlimbs. This coordination is believed to depend on propriospinal axons in dorsolateral and ventral funiculi that interconnect the CPGs (Bem et al., 1995) and on bulbospinal systems, including reticulospinal and vestibulospinal axons (Rossignol and Drew, 1985). Functional locomotion requires, in addition, weight support and postural stability, which depend on several descending pathways (Jankowska and Edgley, 1993; Macpherson et al., 1997). The intact system is sufficiently adaptable to allow normal adult rats to fulfill all the requirements of our tasks virtually without falls or other errors. 


\section{Distribution of serotonin immunoreactivity in cross section caudal to transplant}
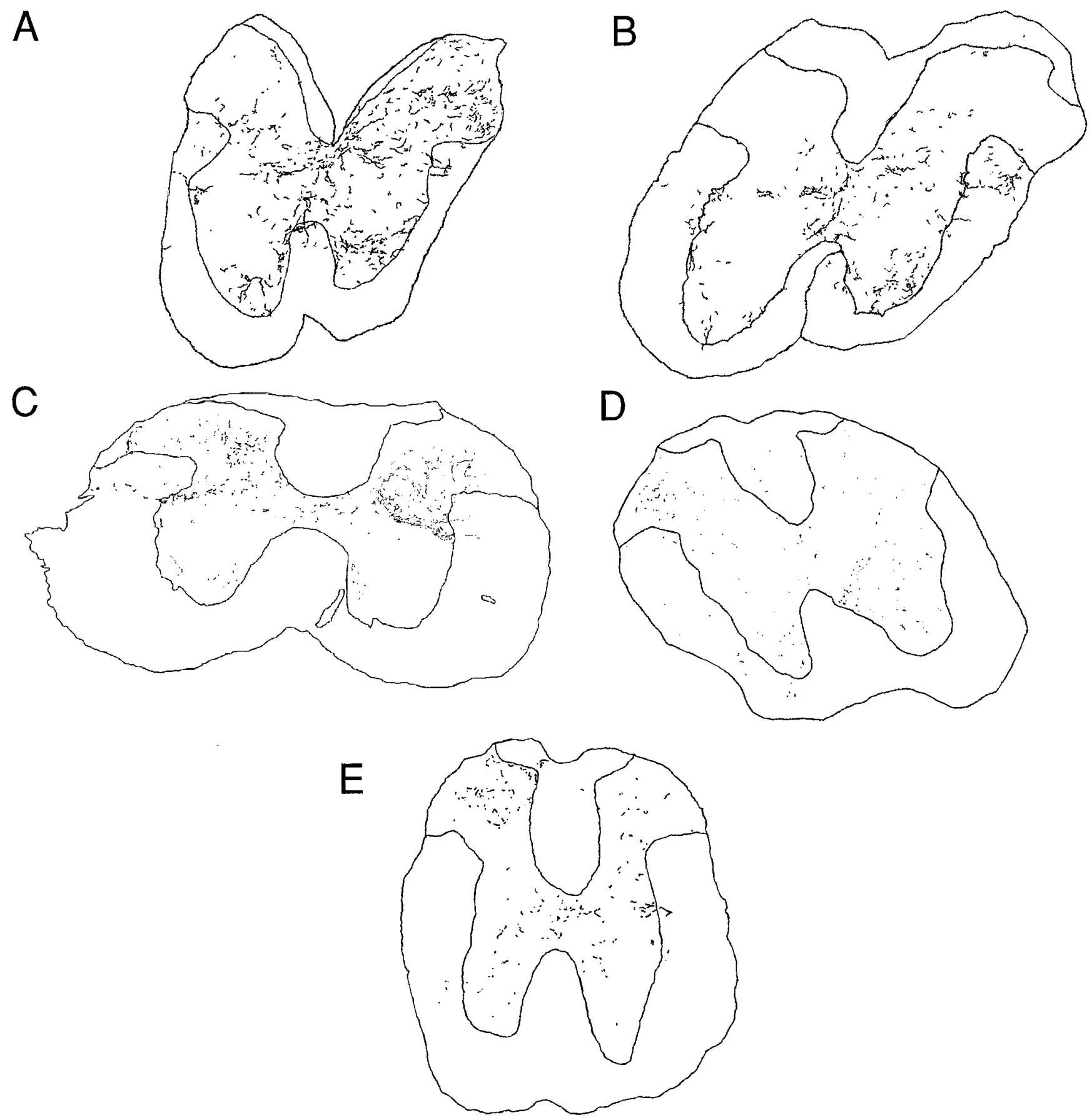

Figure 7. Distribution of $5 \mathrm{HT}$ immunostaining in the segment caudal to the block containing the transplant in five animals. $5 \mathrm{HT}$ immunoreactivity in four animals with good treadmill locomotion: $A$, TP20 (88\% weight-suppported steps); $B$, TP19 (80\%); $C$, TP14 (73\%); $D$, TP 24 (67\%); $E$, 5HT immunoreactivity in animal with poor locomotion (TP21, 38\%). Note the variability of staining in the transplant animals that performed well: primarily dorsally in TP20, ventrally in TP19, both dorsally and ventrally but asymmetrically in TP14. Staining is sparse in TP24. Note staining distributed both dorsally, ventrally, and symmetrically in the poorly performing TP21. 


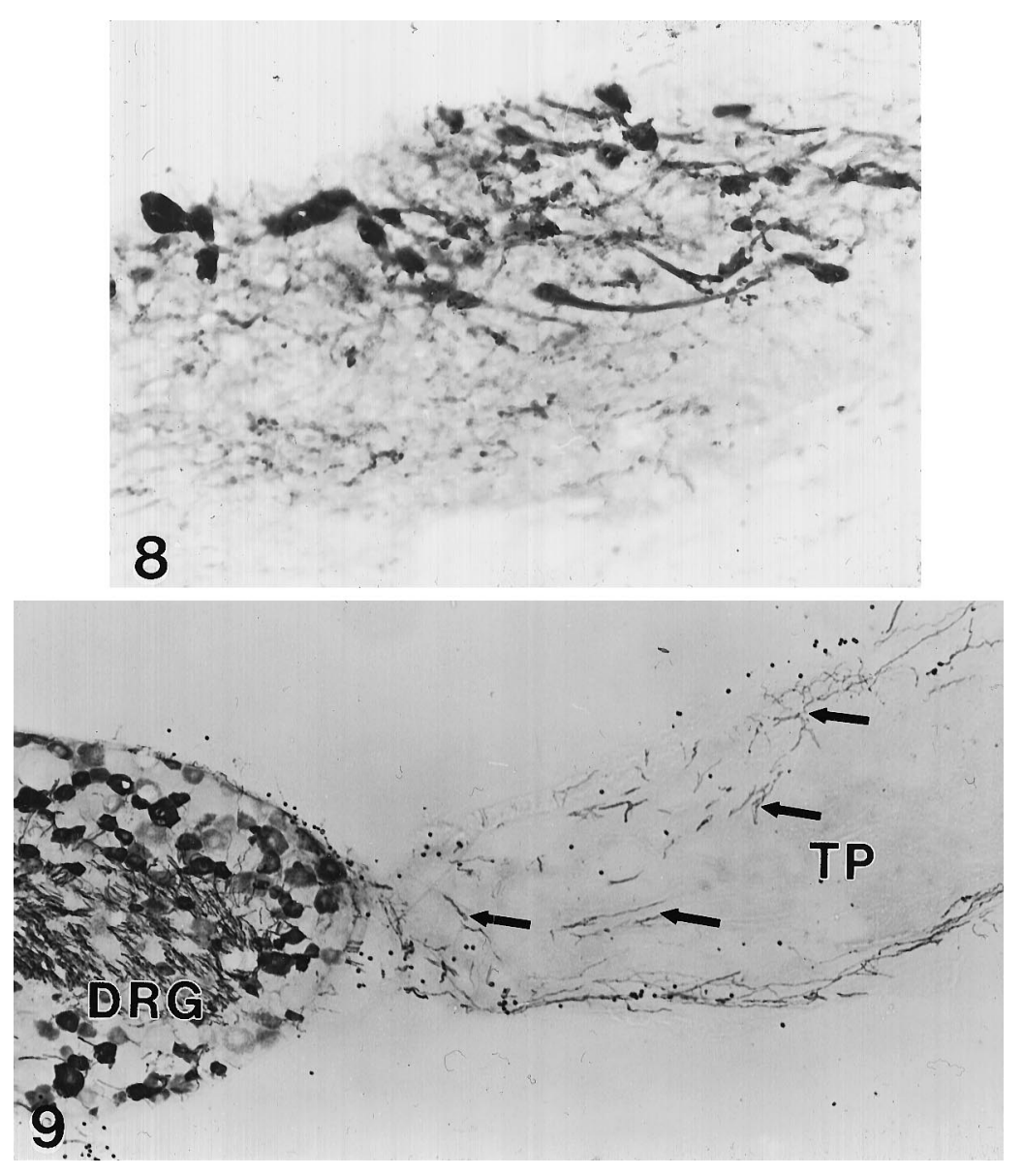

Figure 8. Top. 5HT immunostaining in the transplant shows immunoreactive cells and axons. $100 \times$ magnification.

Figure 9. Bottom. CGRP immunostaining of dorsal root ganglion cells $(D R G)$ and dorsal root axons (arrow) in transplant tissue $(T P) .60 \times$ magnification.

\section{Spinal rats}

The performance of the spinal rats demonstrates the locomotor capacity of the lumbosacral spinal cord isolated from descending input from birth. Consistent with the idea that bipedal stepping requires only an initiating stimulus and an intact $\mathrm{CPG}$, we observed that spinalized rats exhibited reflex hindlimb locomotion when their bodies were supported and their hindlimbs contacted a moving treadmill surface. Control, spinal, and transplant rats showed few differences in this type of reflex locomotion, aside from the tendency of both groups of operated animals to step using the dorsal surface of the hindfoot. Some of our observations indicated that the spinal cord transected in newborns can accomplish aspects of locomotor function that in normal adult rats depend on descending systems. For example, we observed, as have others (Weber and Stelzner, 1977), that spinalized newborn rats may develop hindquarter support that permits overground locomotion in the open field. The mechanism by which some adults spinalized as neonates develop this performance without descending input is unknown. One possibility is that during spontaneous overground locomotion, movement begun by the forelimbs leads to extension of the hindlimbs, which produces sufficient proprioceptive input to the lumbar CPG to induce support of hindquarters and hindlimb locomotion. Proprioceptive information related to hip extension is known to be of particular importance in initiating the transition from stance to swing (Pearson and Duysens, 1976; Grillner and Rossignol, 1978). An additional compensatory mechanism with the potential to initiate locomotion may be provided by proprioceptive information passed across the transection site by the linked mechanics of the body (C. Smeraski and S. Giszter, unpublished observations; S. Giszter and W. Kargo unpublished observations). The same sources may maintain weight support and postural stability once locomotion has begun. There is also evidence that motor cortex organization is modified to different extents in animals spinalized as neonates, which may contribute to the greater function seen in some of these animals (Kargo et al., 1996) (S. Giszter and W. Kargo, unpublished observations). Biochemical and morphological changes that have been described in the injured spinal cord may also heighten the excitability of the lumbosacral segments and contribute to behavioral compensation after the loss of descending systems. These mechanisms include denervation supersensitivity (Roedet et al., 1995; Adipudi et al., 1996) and axonal sprouting by interneurons (Wang et al., 1991a,b) and dorsal root afferent fibers (Stelzner et al., 1979). Training can also improve function (Smith et al., 1982; Lovely et al., 1986; Muir and Steeves, 1997), perhaps in part by eliciting changes in stretch reflex activity that depend on mechanisms intrinsic to the spinal cord itself (Wolpaw and Carp, 1993). There is also evidence, however, that intensive training paradigms can modify neural structures in ways that may have deleterious effects (Jones and Schallert, 1994; Kozlowski et al., 1996).

Our results show that the potential of these mechanisms to compensate for the loss of descending input is limited. As the spinal rats mature and become heavier, the hindlimbs are less able to support the hindquarters, and the frequency of weightsupported steps and the quality of overground locomotion de- 
Figure 10. Photomicrographs from sections taken from TP25, an animal that received a transplant as a neonate and whose cord was retransected as an adult. $a$, Monocyte-derived cells stained with OX42 at interface between transplant and host in the retransected spinal cord. $100 \times$ magnification. $b$, Adjacent cresyl violet-stained section. Rectangle indicates field shown in a. Double arrows indicate site of second transection, which is rostral to the transplant. Single arrow indicates caudal border between transplant and host. Note dorsal root $(D R)$. $40 \times$ magnification.

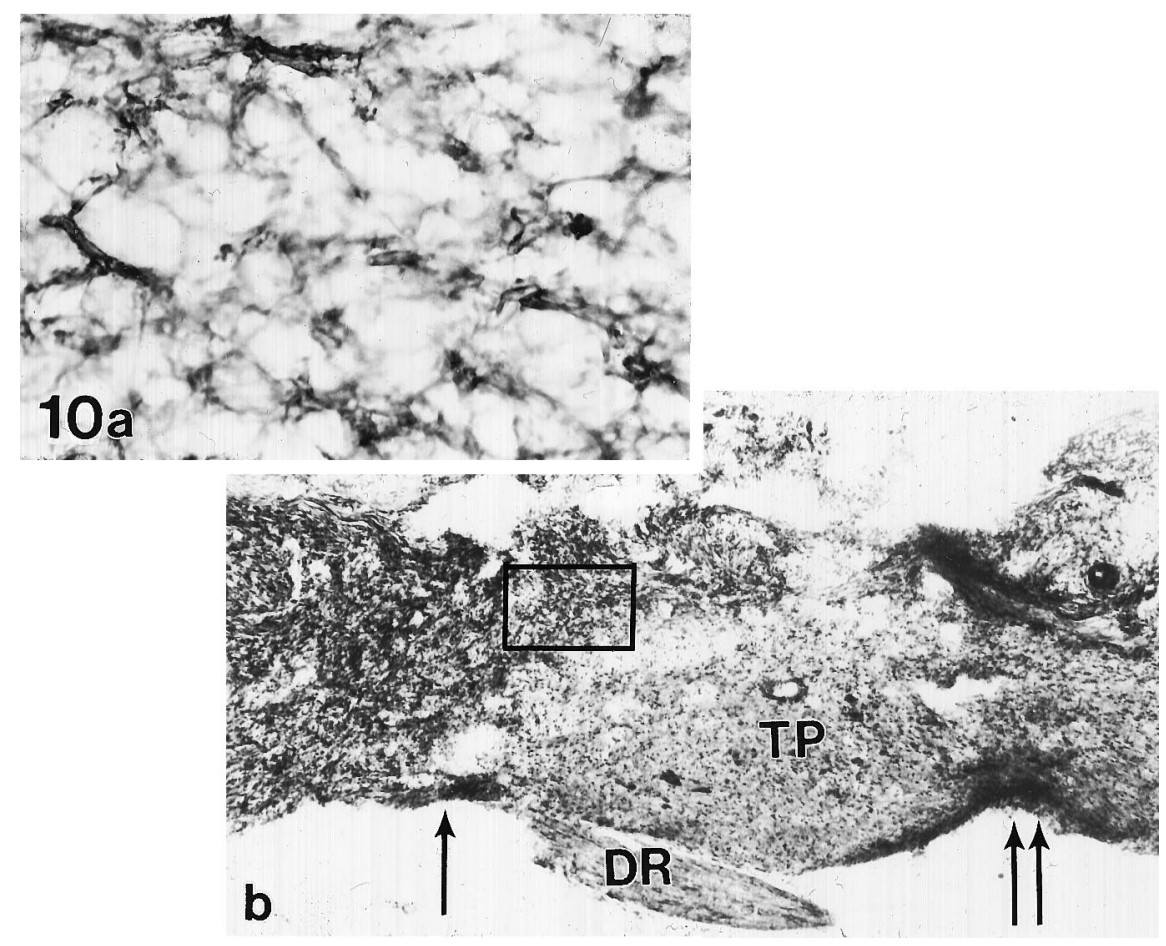

crease. Because transplant and spinal animals showed similar patterns of weight gain, and both gained less weight than their normal littermates, the poor hindquarter weight support of spinal compared with transplant animals cannot be attributed to size alone. Most of our spinal rats also showed relatively few linked weight-supported hindlimb steps during quadrupedal treadmill locomotion; instead they tended to locomote using forelimbs only. This is in contrast with the results of Edgerton's group (Zhang et al., 1994), whose more intensive and interactive training protocol may account for the better hindlimb weight support. Other elements important in locomotion, including postural adjustments to perturbations of center of mass and coordination between forelimbs and hindlimbs, were absent in most spinal rats. Spinal rats were usually unable to complete tasks emphasizing interlimb coordination and postural adjustments, e.g., stair descent and crossing a horizontal ladder. An analysis of corrective responses in cats suggested that although some strategies are organized at the spinal level, the loss of facilitatory descending input accounts for the failure of spinal animals to make appropriate corrections (Hiebert et al., 1994).

\section{Transplant rats}

The locomotor performance of most rats with transplants exceeded that of most spinal rats in the tasks that we examined. Those animals that performed well on the treadmill also performed well, although with fewer weight-supported steps, on the more difficult tasks. Moreover, the final decrement in function after retransection in transplants rats, unlike spinal rats, was substantial. In contrast with most spinal rats, transplant rats used linked weight-supported steps with postural adjustments adequate to maintain body support and coordination between forelimbs and hindlimbs sufficient not only to carry out quadrupedal locomotion on a treadmill but also to cross runways and descend stairs. Surprisingly, at least some of the transplant rats showed improved intralimb and interlimb coordination, foot placement, and balance on the more com- plex tasks of crossing a narrow runway and staircase descent than during overground locomotion on a wide runway. The pattern of performance of transplant rats on more complex tasks, particularly the striking difference between locomotion across wide and narrow runways, suggested that rats used different strategies of motor control, and perhaps different pathways, to accomplish tasks of varying complexity. One possibility consistent with these behavioral results is that transplants allow descending systems to access spinal circuits caudal to the transection and that these connections are recruited when communication between the rostral modules of the spinal cord or brain and lumbar circuits is necessary for finer control. Our observations that aspects of performance that are believed to depend on descending systems, including forelimb-hindlimb coordination, were also enhanced by transplants further support this idea. The difficulty that transplanted rats had in targeting weight-supported steps on ladder rungs, however, suggests that the extent of descending input is limited. Inadequate sensory feedback for the control of the hindlimbs may also contribute to the failure in precise foot placement necessary for ladder crossing.

The mechanisms by which transplants enhance locomotor performance remain a matter of speculation. We observed that transplanted tissue survived in the transection site, although transplant size and extent of integration with the host were variable. A mechanism consistent with our behavioral results is that transplants allow descending systems to influence the caudal spinal cord either directly by growth across the lesion site or indirectly by the formation of relays with donor neurons. 5HT staining caudal to transplants that in some cases could have derived from cell bodies within the host brainstem and in other cases from cell bodies within the transplants is consistent with both direct growth and relay formation. Growth of serotonergic and other supraspinal systems through transplants placed into injured newborn spinal cord has been reported previously (Bregman, 1987); however, 
the patterns of 5HT staining and the extent to which the transplants integrated with the host showed little direct relation to the level of motor functions that developed. The connections established between transplant and host in this series of animals are likely to be variable and rather modest. The impact of even a small amount of descending input may be amplified, however, if it acts in concert with compensatory mechanisms that have been described within the lumbar spinal cord after injury. A redundancy in pathways and in mechanisms for organizing complex motor end points may permit development of function with only modest regeneration. These anatomical results, however, raise the possibility that a major effect of the transplanted tissue is via an indirect or trophic function that is exerted on the lumbar spinal cord and acts to keep alive axotomized host neurons (Bregman and Kunkel-Bagden, 1988; Himes et al., 1994; Mori et al., 1997).

When data from operated (spinal and transplant) rats are pooled, a bimodal distribution of performance for each of the tests is seen. Animals that performed well on one test also performed well on the other tests in our series, although the performance declined markedly on the most difficult task of horizontal ladder crossing. The bimodal distribution suggests a threshold effect for the ability of an animal to develop hindlimb weight support sufficient to permit locomotion. Several factors may contribute to crossing the threshold; our data suggest that the presence of a transplant increases the liklihood that an animal will walk, but other factors that we examined in this study, e.g., the body weight, sibling relationship, or the distribution and amount of regeneration of serotonergic fibers in caudal spinal cord, did not show a clear relationship to the performance in these tasks. Obviously the contributions of other changes in the organization of the nervous system induced by neonatal transections and transplants, including regeneration of other systems and compensatory modifications of spared tissue, need to be examined. We conclude that the several mechanisms by which transplants placed in neonatal rats permit repair of injured spinal cord, including axonal regeneration, rescue of axotomized neurons destined to die, and trophic effects on remaining circuitry, improve the substrate through which compensatory mechanisms operate and thus permit development of improved motor control.

\section{REFERENCES}

Adipudi V, Croul S, Moul J, Aloyo V, Simansky KJ, Battisti W, Murray M (1996) 5HT2c receptor densities in the spinal cord of normal and spinal transected rats. Soc Neurosci Abstr 22:1776.

Barbeau H, Rossignol S (1987) Recovery of locomotion after chronic spinalization in the adult cat. Brain Res 412:84-95.

Bem T, Gorska T, Majczyniski H, Zmyslowski W (1995) Different patterns of fore-hindlimb coordination during overground locomotion in cats with ventral and lateral spinal lesion. Exp Brain Res 104:70-80.

Blight AR, DeCrescito V (1986) Morphometric analysis of experimental spinal cord injury in the cat: the relations of injury intensity to survival of myelinated axons. Neuroscience 19:321-341.

Bregman BS (1987) Spinal cord transplants permit the growth of serotonergic axons across the site of neonatal spinal cord transection. Dev Brain Res 34:265-279.

Bregman BS, Reier PJ (1986) Neural tissue transplants rescue axotomized rubrospinal cells from retrograde death. J Comp Neurol 244:86-95.

Bregman BS, Kunkel-Bagden E (1988) Effects of target and non-target transplants on neuronal survival and axonal elongation after injury to the developing spinal cord. Prog Brain Res 78:205-211.

Cazalets JR, Sqalli-Houssani Y, Clarac F (1994) GABA-ergic innactivation of the central pattern generator for locomotion in the isolated neonatal rat spinal cord. J Physiol (Lond) 474:173-181.
Dunnett SB, Bjorklund A (1994) Mechanisms of function of neural grafts in the injured brain. In: Functional neural transplantation (Dunnett SB, Bjorklund A, eds), pp 531-567. New York: Raven.

Edgerton VR, Roy RR, Hodgson JA, Prober RJ, De Guzman CP, de Leon R (1992) Potential of adult mammalian lumbosacral spinal cord to execute and acquire improved locomotion in the absence of supraspinal input. J Neurotrauma 9:110-127.

Goldberger ME, Bregman BS, Vierck C, Brown M (1990) Criteria for assessing recovery of function after spinal cord injury: behavioral methods. Exp Neurol 107:113-117.

Grillner S, Rossignol S (1978) On the initiation of swing phase of locomotion of chronic spinal cats. Brain Res 146:260-277.

Hiebert GW, Gorassini MA, Jiang W, Prochazka A, Pearson KG (1994) Corrective responses to loss of ground support during walking. II. Comparison of intact and chronic spinal cats. J Neurophysiol 71:611-622.

Himes BT, Goldberger ME, Tessler A (1994) Grafts of fetal central nervous tissue rescue axotomized Clarke's nucleus neurons in adult and neonate operates. J Comp Neurol 339:117-131.

Howland DR, Bregman BS, Goldberger ME (1995a) The development of quadrupedal locomotion in kittens. Exp Neurol 135:93-107.

Howland DR, Bregman BS, Tessler A, Goldberger ME (1995b) Development of locomotor behavior in the spinal kitten. Exp Neurol 135:108-122.

Howland DR, Bregman BS, Tessler A, Goldberger ME (1995c) Transplants enhance locomotion in neonatal kittens whose spinal cords are transected. Exp Neurol 135:123-145.

Itoh Y, Waldeck RF, Tessler A, Pinter MJ (1996) Regenerated dorsal root fibers form funtional synapses in embryonic spinal cord transplants. J Neurophysiol 76:1236-1245.

Iwashita Y, Kawaguchi S, Murata M (1994) Restoration of function by replacement of spinal cord segments in the rat. Nature 367:167-170.

Jankowska E, Edgley S (1993) Interaction between pathways controlling posture and gait at the level of spinal interneurones in the cat. Prog Brain Res 97:161-170.

Jones TA, Schallert T (1994) Use-dependent growth of pyramidal neurons after neocortical damage. J Neurosci 14:2140-2152.

Kargo W, Davis MR, Murray M, Giszter SF (1996) Organization of cortical motor representation in normal, transect and transplant rats. Soc Neurosci Abstr 26:572.

Kiehn O, Kjaerulff O (1996) Spatiotemporal characteristics of 5-HT and dopamine-induced rhythmic hindlimb activity in the in vitro neonatal rat. J Neurophysiol 75:1472-1482.

Kozlowski DA, James DC, Schallert T (1996) Use-dependent exaggeration of neuronal injury after unilateral sensorimotor cortex lesions. J Neurosci 16:4776-4786.

Kudo N, Ozaki S, Yamada T (1991) Ontogeny of rhythmic activity in the spinal cord of the rat. In: Neurobiological basis of human locomotion (Shimamura M, Grillner S, Edgerton VR, eds), pp 127-137. Tokyo: Japan Scientific Societies.

Kunkel-Bagden E, Dai H-N, Bregman BS (1993) Methods to assess the development and recovery of locomotor function after spinal cord injury in rats. Exp Neurol 119:153-164.

Loveley RG, Gregor RJ, Roy RR, Edgerton VR (1986) Effects of training on the recovery of full-weight bearing stepping in the adult spinal cat. Exp Neurol 92:421-435.

Macpherson JM, Fung J, Jacob R (1997) Postural orientation, equilibrium, and the spinal cord. Adv Neurol 72:227-232.

Mori F, Himes BT, Kowada M, Murray M, Tessler A (1997) Fetal spinal cord transplants rescue some axotomized rubrospinal neurons from retrograde cell death in adult rats. Exp Neurol 143:45-60.

Muir GD, Steeves JD (1997) Sensorimotor stimulation to improve locomotor recovery after spinal cord injury. Trends Neurosci 20:72-77.

Pearson, K, Duysens J (1976) Function of segmental reflexes in the control of stepping in cockroaches and cats. In: Neural control of locomotion (Herman RM, Grillner S, Stein PSG, Stuart DG, eds), pp 587-615. New York: Plenum.

Pratt CA, Fung J, MacPherson JM (1994) Stance control in the chronic spinal cat. J Neurophysiol 71:1981-1985.

Roedet C, Gimenez y Ribotta M, Privat A, Feuerstein C, Savasta M (1995) Intraspinal noradrenaline rich implants reverse the increase of a-1 adrenoceptor densities caused by complete spinal cord transection or selective chemical denervation. Brain Res 677:1-12.

Rossignol S, Drew T (1985) Interactions of segmental and supersegmen- 
tal inputs with the spinal pattern generator of locomotion. In: Feedback and motor control (Barnes WJP, Gladden MH, eds), pp 355-377. London: Croomhelm.

Smith JL, Smith LA, Zernicke RF, Hoy M (1982) Locomotion in exercised and non-exercised cats cordotomized at two or twelve weeks of age. Exp Neurol 76:393-413.

Stelzner DJ, Ershler WB, Weber ED (1975) Effects of spinal transection in neonatal and weanling rats: survival of function. Exp Neurol 46:156-177.

Stelzner DJ, Weber ED, Prendergast J (1979) A comparison of the effect of midthoracic hemisection in neonatal and weanling rats on the distribution and density of dorsal root axons of the lumbosacral spinal cord of the rat. Brain Res 172:407-426.
Wang SD, Goldberger ME, Murray M (1991a) Normal development and the effects of early rhizotomy on spinal systems in the rat. Dev Brain Res 64:57-69.

Wang SD, Goldberger ME, Murray M (1991b) Plasticity of spinal systems after unilateral lumbosacral dorsal rhizotomy in the adult rat. J Comp Neurol 304:555-568.

Weber ED, Stelzner DJ (1977) Behavioral effects of spinal cord transection in the developing rat. Brain Res 125:241-255.

Wolpaw JR, Carp JS (1993) Adaptive plasticity in the spinal cord. Adv Neurol 59:163-174.

Zhang AA, Kirkpatrick G, Zhong VH, Nguyen VT, Dobkin BH, Edgerton VR (1994) Cinematographic analysis of hindlimb stepping in spinal rats. Soc Neurosci Abstr 24:571. 